\title{
Multiclass classification with bandit feedback using adaptive regularization
}

\author{
Koby Crammer • Claudio Gentile
}

Received: 28 August 2011 / Accepted: 5 September 2012 / Published online: 24 October 2012

(C) The Author(s) 2012

\begin{abstract}
We present a new multiclass algorithm in the bandit framework, where after making a prediction, the learning algorithm receives only partial feedback, i.e., a single bit indicating whether the predicted label is correct or not, rather than the true label. Our algorithm is based on the second-order Perceptron, and uses upper-confidence bounds to trade-off exploration and exploitation, instead of random sampling as performed by most current algorithms. We analyze this algorithm in a partial adversarial setting, where instances are chosen adversarially, while the labels are chosen according to a linear probabilistic model which is also chosen adversarially. We show a regret of $\mathcal{O}(\sqrt{T} \log T)$, which improves over the current best bounds of $\mathcal{O}\left(T^{2 / 3}\right)$ in the fully adversarial setting. We evaluate our algorithm on nine real-world text classification problems and on four vowel recognition tasks, often obtaining state-of-the-art results, even compared with non-bandit online algorithms, especially when label noise is introduced.
\end{abstract}

Keywords Online learning · Upper confidence bound · Regret

\section{Introduction}

Consider a book recommendation system. Given a customer's profile, it recommends a few possible books to the user, with the aim of choosing books that the user will like and eventually purchase. Typical feedback in such a system is the actual action of the user, or specifically what books has she bought, if any. The system cannot observe what would have been the user's actions had other books got recommended.

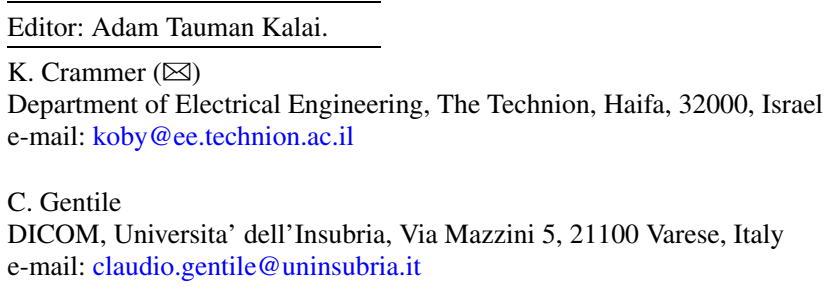


Generally, such problems are referred to as learning with partial feedback. Unlike the full information case, where the system or the learning algorithm knows the outcome of each possible response, e.g., the user's action for each and every possible book recommended, in the partial setting, the system observes the response only to very limited options and, in particular, the option that was actually recommended.

We consider an instantiation of this problem in the multiclass prediction problem within the online bandit setting. Learning is performed in rounds. On each round the algorithm receives an instance and outputs a label from a finite set of size $K$. It then receives a single bit indicating whether the predicted label is correct or not, which the algorithm uses to update its internal model and proceed to the next round. Note that for rounds where the feedback indicates wrong prediction, the algorithm's uncertainty about the true label for that instance is almost not reduced, since the number of alternatives is only reduced from $K$ to $K-1$. Hence the algorithm needs somehow to follow an exploration-exploitation strategy.

Related work Our algorithm trades-off exploration and exploitation via upper-confidence bounds, in a way that is somewhat similar to the work of Auer (2003) and Dani et al. (2008). Yet, the result most closely related to our work is the Banditron algorithm of Kakade et al. (2008), which builds on the immortal Perceptron algorithm. Kakade et al. (2008) investigated this problem in an online adversarial setting, and showed a $\mathcal{O}\left(T^{2 / 3}\right)$ bound on the regret compared to the hinge loss of a linear-threshold comparator. Wang et al. (2010) extended their results to a more general potential-based framework for online learning.

Multiclass classification with bandit feedback can be seen as a multi-armed bandit problem with side information. Relevant work within this research thread includes the GreedyEpoch algorithm analyzed by Langford and Zhang (2007), where a $\mathcal{O}\left(T^{2 / 3}\right)$ regret bound has been proven under i.i.d. assumptions, yet covering more general learning tasks than ours. We are aware of at least three more papers that define multi-armed bandit problems with side information, also called bandits with covariates: Wang et al. (2005), Lu et al. (2010), Rigollet and Zeevi (2010). However, the models in these paper are very different from ours, and not easily adapted to our multiclass problem. Another paper somewhat related to this work is by Walsh et al. (2009), where the authors adopt a similar linear model as ours (and similar mathematical tools) in a setting where an online prediction algorithm is allowed to sometimes answer "I don't know" (the so-called KWIK setting). A direct adaptation of their results to our multiclass setting is not straightforward. It is, however, easy to adapt their work to the binary (non-bandit) setting. In this case, their algorithm is shown to approximate the Bayes predictor with a convergence rate of the form $T^{-1 / 4}$. This result is significantly inferior to our convergence results, and it seems to hold only in the finite-dimensional case. We do not expect any multiclass adaptation of their results to lead to improved convergence rates.

Two papers (Valizadegan et al. 2011; Hazan and Kale 2011) more closely related to our work became available after our paper was submitted; we briefly discuss these contributions in Sect. 8.

Our results We study a setting related to the one by Kakade et al. (2008) and Wang et al. (2010), in which we assume a probabilistic linear model over the labels, although the instances are chosen by an adaptive adversary. We develop a bandit algorithm building on the 2nd-order Perceptron algorithm using the correlation matrix maintained by the algorithm to estimate uncertainty in prediction. We show regret bounds of $\mathcal{O}(\sqrt{T} \log T)$, which are essentially optimal in this setting (up to log factors). We evaluate our algorithms on nine real-world text classification tasks and four vowel recognition tasks which vary in size, feature complexity and number of labels. We show that our algorithm always outperforms the 
Banditron algorithm. In fact, on a few datasets our algorithm also outperforms Perceptron and 2nd-order Perceptron working with full information labels, especially when label noise is induced. Finally, we sketch an extension of our results to the case when the labels have an arbitrary distribution, and an approximation error to our linear noise model has to be taken into account.

\section{Multiclass bandit online learning}

Standard online learning with full information is performed in rounds. On round $t$ the algorithm receives an instance $\boldsymbol{x}_{t} \in \mathbb{R}^{d}$ to be classified, and predicts a label $\hat{y} \in\{1 \ldots K\}$. It receives the true label $y_{t}$, updates its internal model, and is ready for the next round.

The algorithm we present employs linear models. The algorithm maintains $K$ weight vectors $\boldsymbol{w}_{i} \in \mathbb{R}^{d}$, for $i=1, \ldots, K$. Given an instance $\boldsymbol{x}_{t}$, the algorithm computes a score associated with each of the $K$ classes, defined by $\boldsymbol{w}_{i}^{\top} \boldsymbol{x}_{t}$, and outputs a prediction to be the label with the highest score, that is,

$$
\tilde{y}_{t}=\arg \max _{i=1, \ldots, K} \boldsymbol{w}_{i}^{\top} \boldsymbol{x}_{t} .
$$

We emphasize that two quantities are considered: the label with maximal score $\tilde{y}_{t}$ defined in Eq. (1) (this quantity is internal to the algorithm), and the label that is actually output by the algorithm, denoted by $\hat{y}_{t}$. In the full-information case, most algorithms just output their prediction, that is we have $\hat{y}_{t}=\tilde{y}_{t}$. In this paper, we focus on the partial information setting, also known as the bandit setting. Here, after the algorithm makes a prediction, it does not receive the correct label $y_{t}$ but only a single bit $M_{t}$ indicating whether its output $\hat{y}_{t}$ was correct or not, i.e.,

$$
M_{t}=\left\{y_{t} \neq \hat{y}_{t}\right\},
$$

where $\{A\}$ is 1 if the predicate $A$ is true, and 0 otherwise.

Since learning algorithms receive only very limited feedback there is a natural tradeoff between exploration and exploitation. On the one hand, the algorithm should output the best scoring label $\tilde{y}_{t}=\arg \max _{i=1 \ldots K} \boldsymbol{w}_{i}^{\top} \boldsymbol{x}_{t}$, this step being called exploitation. Yet, it may be the case that the model used at some point will not perform well (for example, the initial model), and thus the algorithm will make many mistakes and most of its feedback will indicate that the output is not correct, that is $M_{t}=1$. This feedback is almost useless, as there is still uncertainty about the true label (one of $K-1$ options remain). On the other hand, the algorithm may perform exploration and output another label to get useful feedback.

The Banditron algorithm implements one approach to exploration-exploitation tradeoff. From time to time, the algorithm outputs another label than its prediction $\tilde{y}$. The Banditron chooses such examples at random with some probability $\gamma$, and then it chooses a random label uniformly with probability $1 / K$. This approach ignores few aspects of the state. First, it ignores the specific input $\boldsymbol{x}_{t}$ to be labeled, although some inputs may be classified well by the current model, and others may not. For example, in a deterministic setting where each instance vector is associated with only a single label, if an example is repeated few times, and the algorithm receives feedback about its true label, then it should output the best scoring label rather than sampling a label. Second, it ignores the difference in score values $\boldsymbol{w}_{1}^{\top} \boldsymbol{x}_{t}, \ldots, \boldsymbol{w}_{K}^{\top} \boldsymbol{x}_{t}$. For example, it may be the case that two of the score values are very large compared to the others; then it makes sense to output only one of the two, rather than sampling from the entire label set. Third, it ignores the example index $t$, as it is reasonable to assume that as the algorithm learns more it needs to explore less. 
An alternative approach, which we employ in this work, is to maintain additional confidence information about the predictions. Specifically, given an input $\boldsymbol{x}_{t}$, the algorithm not only computes score values, but also non-negative uncertainty values for these scores, denoted by $\epsilon_{i, t}$. Intuitively, high values of $\epsilon_{i, t}$ indicate that the algorithm is less confident in the value of the score $\boldsymbol{w}_{i}^{\top} \boldsymbol{x}_{t}$. Given a new example, the algorithm outputs the label with the highest upper confidence bound (UCB), computed as the sum of score and uncertainty, $\hat{y}_{t}=\arg \max _{i}\left(\boldsymbol{w}_{i}^{\top} \boldsymbol{x}_{t}+\epsilon_{i, t}\right)$. Intuitively, a label $\hat{y}$ is output by the algorithm if either its score is high or the uncertainty in predicting it is high, and there is need to obtain information about it. Specifically, our algorithm maintains a positive semidefinite matrix per label, $A_{i, t} \in \mathbb{R}^{d \times d}$. Given an input instance $\boldsymbol{x}_{t}$ to be classified, we define the confidence intervals to be $\epsilon_{i, t}^{2}=\eta_{t} \boldsymbol{x}_{t}^{\top} A_{i, t}^{-1} \boldsymbol{x}_{t}$ for some scalar $\eta_{t}$ which is used to tradeoff the exploration and exploitation. The matrices $A_{i, t}$ (or their inverses) are used to measure uncertainty in the score, and input examples are used to update them as well as the parameters of the scoring function $\boldsymbol{w}_{i}$ 's.

We now describe the specific model we use to motivate our algorithm, and later analyze it. In Sect. 4.2 we sketch an extended analysis that works under more general assumptions and show that the regret is larger and depends on how much the general model is different from the proposed one.

Our setting is slightly less adversarial than the one considered in Kakade et al. (2008), Wang et al. (2010). In particular, we assume the following parametric model for the multiclass labels: ${ }^{1}$ we assume that the labels of an example $\boldsymbol{x}_{t}$ are generated according to the following probabilistic model,

$$
\mathbb{P}\left(y_{t}=i \mid \boldsymbol{x}_{t}\right)=\frac{\alpha+\boldsymbol{u}_{i}^{\top} \boldsymbol{x}_{t}}{\alpha+1},
$$

for some $K$ vectors $\boldsymbol{u}_{1}, \ldots, \boldsymbol{u}_{K} \in \mathbb{R}^{d}$, and a scalar $\alpha \in(-1,1]$. The model is well defined if, for all $\boldsymbol{x} \in \mathbb{R}^{d}$ chosen by the adversary, we have

$$
\sum_{i=1}^{K} \boldsymbol{u}_{i}^{\top} \boldsymbol{x}=\alpha+1-K \alpha \quad \text { and } \quad-\alpha \leq \boldsymbol{u}_{i}^{\top} \boldsymbol{x} \quad \forall i .
$$

(Notice that this implies $\boldsymbol{u}_{i}^{\top} \boldsymbol{x} \leq 1$ for all $i$ ). For simplicity we also assume $\left\|\boldsymbol{x}_{t}\right\|=1$ for all $t$. Given some weight vectors $\boldsymbol{u}_{i}$ for $i=1, \ldots, K$ and an input $\boldsymbol{x}$, the constraints of $\frac{\alpha+\boldsymbol{u}_{i}^{\top} \boldsymbol{x}}{\alpha+1}$ being a probability vector are enforced by projecting these weight vectors onto the constraints $\sum_{i=1}^{K} \boldsymbol{u}_{i}^{\top} \boldsymbol{x}=\alpha+1-K \alpha$ and $-\alpha \leq \boldsymbol{u}_{i}^{\top} \boldsymbol{x}$ for $i=1, \ldots K$, as defined in (6). In Sect. 3.1 below we show how to compute this projection efficiently.

Intuitively, $\alpha$ quantifies the closeness of the $K$ tasks according to model (2). On the one hand, the closer $\alpha$ gets to -1 the more the scores $\boldsymbol{u}_{i}^{\top} \boldsymbol{x}$ are forced to be close to each other (i.e., $\boldsymbol{u}_{i}^{\top} \boldsymbol{x} \approx 1$ for all $i$, independent of $\boldsymbol{x}$ ). On the other hand, setting $\alpha=1$ yields $\mathbb{P}\left(y_{t}=i \mid \boldsymbol{x}_{t}\right)=\frac{1+\boldsymbol{u}_{i}^{\top} \boldsymbol{x}_{t}}{2}$ under the constraints $-1 \leq \boldsymbol{u}_{i}^{\top} \boldsymbol{x} \leq 1$ and $\sum_{i=1}^{K} \boldsymbol{u}_{i}^{\top} \boldsymbol{x}=2-K$. This choice allows the probability mass to be concentrated on the $i$-th label by setting $\boldsymbol{u}_{i}^{\top} \boldsymbol{x}=1$ for some $i$ and $\boldsymbol{u}_{i}^{\top} \boldsymbol{x}=-1$ otherwise. Two natural choices of $\alpha$ are $\alpha=\frac{1}{K-1}$ and $\alpha=0$. The former yields

$$
\mathbb{P}\left(y_{t}=i \mid \boldsymbol{x}_{t}\right)=\frac{1+\boldsymbol{u}_{i}^{\top} \boldsymbol{x}_{t}(K-1)}{K}
$$

\footnotetext{
${ }^{1}$ This model is a natural extension of the binary label noise model considered by Cesa-Bianchi et al. (2009) and Dekel et al. (2010).
} 
under the constraints

$$
-\frac{1}{K-1} \leq \boldsymbol{u}_{i}^{\top} \boldsymbol{x} \leq 1 \quad \text { and } \quad \sum_{i=1}^{K} \boldsymbol{u}_{i}^{\top} \boldsymbol{x}=0
$$

which can be satisfied by a constraint that is independent of $\boldsymbol{x}_{t}$, namely, $\sum_{i=1}^{K} \boldsymbol{u}_{i}=0$. The latter choice $\alpha=0$ forces $\left(\boldsymbol{u}_{1}^{\top} \boldsymbol{x}, \ldots, \boldsymbol{u}_{K}^{\top} \boldsymbol{x}\right)$ to be a probability vector. For the sake of our analysis (Sect. 4), we will restrict our attention to the case $\alpha \geq 0$.

We will bound the extent to which the number of prediction mistakes of our learning algorithms exceeds the number of prediction mistakes of the Bayes optimal predictor

$$
b_{t}=b\left(\boldsymbol{x}_{t}\right)=\arg \max _{i=1, \ldots, K} \mathbb{P}\left(y_{t}=i \mid \boldsymbol{x}_{t}\right)=\arg \max _{i=1, \ldots, K}\left(\boldsymbol{u}_{i}^{\top} \boldsymbol{x}_{t}\right)
$$

for this label noise model. In particular, we are aimed to bound from above the cumulative regret

$$
R_{T} \equiv \sum_{t=1}^{T}\left(\mathbb{P}_{t}\left(y_{t} \neq \hat{y}_{t}\right)-\mathbb{P}_{t}\left(y_{t} \neq b\left(\boldsymbol{x}_{t}\right)\right)\right)
$$

with high probability over past $y$ 's, possibly taking into account the internal randomization of the algorithms. In the above expression, $\mathbb{P}_{t}$ denotes the conditional probability $\mathbb{P}\left(\cdot \mid \boldsymbol{x}_{1}, \ldots \boldsymbol{x}_{t}, y_{1}, \ldots y_{t-1}, \sigma_{t-1}\right)$, where it is understood that $\boldsymbol{x}_{t}$ can also be chosen adversarially as a function of past $\boldsymbol{x}$ and $y$, and $\sigma_{t-1}$ is the (possible) internal randomization of the algorithm under consideration up to round $t-1$. Similarly, we denote by $E_{t}[\cdot]$ the conditional expectation $E_{t}\left[\cdot \mid \boldsymbol{x}_{1}, \ldots \boldsymbol{x}_{t}, y_{1}, \ldots y_{t-1}, \sigma_{t-1}\right]$.

Notice that the regret (3) is comparing (expected) cumulative 0/1-loss of the algorithm to (expected) cumulative 0/1-loss of the best offline linear predictor. Hence, our notion of regret is also sharper than the one adopted in Kakade et al. (2008) and Wang et al. (2010), whose regret compares $0 / 1$ loss to hinge loss.

Our algorithm is a variant of the multiclass second-order Perceptron algorithm that maintains at time $t$ a set of $K$ vectors $\boldsymbol{w}_{1, t}, \ldots, \boldsymbol{w}_{K, t} \in \mathbb{R}^{d}$, where $\hat{\Delta}_{i, t}=\boldsymbol{w}_{i, t-1}^{\top} \boldsymbol{x}_{t}$ is intended to approximate $\Delta_{i, t}=\boldsymbol{u}_{i}^{\top} \boldsymbol{x}_{t}$ for all $i$ and $t$. The bandit algorithm also maintains a set of $K$ matrices $A_{i, t}$, which are used to compute a standard upper confidence scheme of the form

$$
\hat{y}_{t}=\arg \max _{i=1, \ldots, K}\left(\hat{\Delta}_{i, t}+\epsilon_{i, t}\right),
$$

where $\epsilon_{i, t}$ is a suitable upper confidence level for class $i$ at time $t$, which is a function of both $\boldsymbol{x}_{t}$ and $A_{i, t}$.

\section{The new bandit algorithm}

Our algorithm, described in Fig. 1, is parameterized by the model parameter $\alpha \in(-1,1]$, assumed to be known. The algorithm maintains, for each class $i=1, \ldots, K$, a weight vector $\boldsymbol{w}_{i} \in \mathbb{R}^{d}$ and a correlation matrix $A_{i} \in \mathbb{R}^{d \times d}$, and operates similarly to 2nd-order (or ridge regression)-like algorithms (Hoerl and Kennard 1970; Azoury and Warmuth 2001; CesaBianchi et al. 2005) (see also, e.g., Strehl and Littman 2008; Crammer et al. 2009b; CesaBianchi et al. 2009; Dekel et al. 2010; Dredze et al. 2008 and references therein). The weight vectors are initialized to zero, and the matrices $A_{i}$ are initialized to $(1+\alpha)^{2}$ times the identity 
Parameter: $\alpha \in(-1,1]$

\section{Initialization:}

$A_{0}=(1+\alpha)^{2} I \in \mathbb{R}^{d K \times d K}, W_{0}=\left(\boldsymbol{w}_{1,0}, \boldsymbol{w}_{2,0}, \ldots, \boldsymbol{w}_{K, 0}\right)=0 \in \mathbb{R}^{d K} ;$

For $t=1,2 \ldots, T$ :

1. Get instance $\boldsymbol{x}_{t} \in \mathbb{R}^{d}:\left\|\boldsymbol{x}_{t}\right\|=1$;

2. Set (see Eq. (5))

$$
W_{t-1}^{\prime}=\operatorname{argmin} d_{t-1}\left(W, W_{t-1}\right)
$$

the min being over $W=\left(\boldsymbol{w}_{1}, \ldots, \boldsymbol{w}_{K}\right) \in \mathbb{R}^{d K}$ :

$$
-\alpha \leq \boldsymbol{w}_{i}^{\top} \boldsymbol{x}_{t} \text { for } i=1, \ldots, K, \sum_{i=1}^{K} \boldsymbol{w}_{i}^{\top} \boldsymbol{x}_{t}=1+\alpha-K \alpha
$$

3. Set $\hat{\Delta}_{i, t}^{\prime}=\boldsymbol{x}_{t}^{\top} \boldsymbol{w}_{i, t-1}^{\prime}, \quad i=1, \ldots, K$;

4. Output $\hat{y}_{t}=\operatorname{argmax}_{i}\left(\hat{\Delta}_{i, t}^{\prime}+\epsilon_{i, t}\right)$, where (see Eq. (9))

$$
\begin{aligned}
\epsilon_{i, t}^{2} & =\left(2 \boldsymbol{x}_{t}^{\top} A_{i, t-1}^{-1} \boldsymbol{x}_{t}\right) \times \eta_{t}, \\
\eta_{t} & =\frac{1}{2}(1+\alpha)^{2}\|U\|_{2}^{2}+\frac{(1+\alpha)^{2}}{2} \sum_{s=1}^{t-1} \boldsymbol{x}_{s}^{\top} A_{\hat{y}_{s}, s}^{-1} \boldsymbol{x}_{s}+9(1+\alpha)^{2} \log \frac{t+4}{\delta}
\end{aligned}
$$

5. Get feedback $M_{t}=\left\{y_{t} \neq \hat{y}_{t}\right\}$;

6. If $M_{t}=1$ then:

6a. with prob. $(1-\alpha) / 2$ set

$$
X_{t}=(0, \ldots, 0, \underbrace{\boldsymbol{x}_{t}}_{\text {position } \hat{y}_{t}}, 0, \ldots, 0)
$$

6b. with prob. $(1+\alpha) / 2$ set

$$
X_{t}=(0, \ldots, 0, \underbrace{-\boldsymbol{x}_{t}}_{\text {position } \hat{y}_{t}}, 0, \ldots, 0)
$$

7. Else $\left(M_{t}=0\right)$ set

$$
X_{t}=(0, \ldots, 0, \underbrace{\boldsymbol{x}_{t}}_{\text {position } \hat{y}_{t}}, 0, \ldots, 0)
$$

8. Update:

$$
\begin{aligned}
& A_{t}=A_{t-1}+X_{t} X_{t}^{\top}, \\
& W_{t}=A_{t}^{-1}\left(A_{t-1} W_{t-1}^{\prime}+X_{t}\right) .
\end{aligned}
$$

Fig. 1 The multiclass bandit algorithm Confidit 
matrix $I$ of size $d$. For brevity, we denote by $A$ a single matrix of size $d K \times d K$ defined to be the block-diagonal matrix $A=\operatorname{diag}\left(A_{1}, A_{2}, \ldots, A_{K}\right)$. We also denote by $W$ the $d K$ dimensional vector which is defined to be the concatenation of the $K$ vectors $\boldsymbol{w}_{i}$. Similarly, $U \in \mathbb{R}^{d K}$ is the concatenation of the $K$ vectors $\boldsymbol{u}_{i}$ defined in (2). We use both notations below, each in turn to simplify the presentation in place.

Our algorithm works in rounds. On round $t$, the algorithm receives the (normalized) instance $\boldsymbol{x}_{t} \in \mathbb{R}^{d}$ and defines the following time- $t$ convex set

$$
C_{t}=\left\{W=\left(\boldsymbol{w}_{1}, \ldots, \boldsymbol{w}_{K}\right) \in \mathbb{R}^{d K}:-\alpha \leq \boldsymbol{w}_{i}^{\top} \boldsymbol{x}_{t}, i=1, \ldots, K, \sum_{i=1}^{K} \boldsymbol{w}_{i}^{\top} \boldsymbol{x}_{t}=1+\alpha-K \alpha\right\}
$$

The reader should observe that for each $t$, set $C_{t}$ includes the parameter space where vectors $\boldsymbol{u}_{i}$ are assumed to live (see text surrounding Eq. (2)). The algorithm then projects the current vector $W_{t-1}$ onto $C_{t}$ yielding $W_{t-1}^{\prime}=\left(\boldsymbol{w}_{1, t-1}^{\prime}, \ldots, \boldsymbol{w}_{K, t-1}^{\prime}\right)$. The projection is performed using the multiclass Mahalanobis distance

$$
d_{t-1}(U, W)=\frac{1}{2}(U-W)^{\top} A_{t-1}(U-W)=\sum_{i=1}^{K} \frac{1}{2}\left(\boldsymbol{u}_{i}-\boldsymbol{w}_{i}\right)^{\top} A_{i, t-1}\left(\boldsymbol{u}_{i}-\boldsymbol{w}_{i}\right) .
$$

This projection can be computed efficiently in time $\mathcal{O}(K \log K)$, the details are given in Sect. 3.1.

The algorithm uses $\boldsymbol{w}_{i, t-1}^{\prime}$ to estimate the score-values $\hat{\Delta}_{i, t}^{\prime}=\boldsymbol{x}_{t}^{\top} \boldsymbol{w}_{i, t-1}^{\prime}$, and the upper confidence prediction $\hat{y}_{t}=\arg \max _{i}\left(\hat{\Delta}_{i, t}^{\prime}+\epsilon_{i, t}\right)$ is output. Upon receiving the binary feedback $M_{t}$, the algorithm performs either a deterministic or a randomized update, depending on the value of $M_{t}$. Specifically, if a mistake has been made $\left(M_{t}=1\right)$ then the algorithm flips a coin with bias $\frac{1+\alpha}{2}$ and goes with the update the vector $X_{t}=\left(0, \ldots, 0, \pm \boldsymbol{x}_{t}, 0, \ldots, 0\right)$ depending on the value of the coin. On the other hand, if no mistake is made in that time step $\left(M_{t}=0\right)$, the associated update vector is $X_{t}=\left(0, \ldots, 0, x_{t}, 0, \ldots, 0\right)$, independent of $\alpha$. In all cases, the nonzero block of $X_{t}$ is in position $\hat{y}_{t}$, i.e., only the $\hat{y}_{t}$ 's predictor gets directly affected by $X_{t}$. The constructed update vectors are used within a standard 2nd-order updating scheme, where matrix $A_{t-1}$ undergoes a rank-one update $A_{t} \leftarrow A_{t-1}+X_{t} X_{t}^{\top}$, and vector $W_{t-1}^{\prime}$ turns to vector $W_{t}$ through an additive update $A_{t} W_{t} \leftarrow A_{t-1} W_{t-1}^{\prime}+X_{t}$. The update is well defined as the matrices $A_{t}$ are positive definite (hence nonsingular), since we initialize $A_{0}$ to be positive definite, and the eigenvalues of the matrices $A_{t}$ are nondecreasing with $t$. We call this algorithm Confidit, for (upper) confidence based bandit algorithm.

The construction of the update vector $X_{t}$ essentially determines the algorithm's behavior: The updating sign, denoted by $\beta_{t}= \pm 1$, of $\boldsymbol{x}_{t}$ within $X_{t}$ acts either as a promoter for class $\hat{y}_{t}$ or a demoter, depending on whether $\beta_{t}$ is positive or negative. First, observe that, if the algorithm makes a mistake, then $\beta_{t}$ is on average equal to $-\alpha$, i.e., $E_{t}\left[\beta_{t} \mid M_{t}=1\right]=-\alpha$. Hence on a mistaken trial we demote (the mistaken) class $\hat{y}_{t}$ only if $\alpha$ is positive. (On the contrary, if $\alpha$ is negative the algorithm deems all class predictors $\Delta_{i, t}$ to be very close to each other, hence promoting one class is somewhat similar to promoting all the other ones. Recall that the projection step forces them to stay very close anyway. This is one of the reasons why we restrict our discussion to nonnegative values of $\alpha$.) Second, it is worth observing that, conditioning only on the past, and setting $p_{t}=\mathbb{P}_{t}\left(M_{t}=0\right)=\frac{\alpha+\Delta_{\hat{t}, t}}{1+\alpha}$, we have 


$$
\begin{aligned}
E_{t}\left[\beta_{t}\right] & =E_{t}\left[\beta_{t} \mid M_{t}=1\right]\left(1-p_{t}\right)+E_{t}\left[\beta_{t} \mid M_{t}=0\right] p_{t} \\
& =E_{t}\left[\beta_{t} \mid M_{t}=1\right]\left(1-p_{t}\right)+p_{t} \\
& =-\alpha\left(1-p_{t}\right)+p_{t} \\
& =\Delta_{\hat{y}_{t}, t} .
\end{aligned}
$$

Hence this expectation is positive if and only if $\Delta_{\hat{y}_{t}, t}>0$. One way of stating this is that on any given time step (mistaken or not), $\hat{\Delta}_{\hat{y}_{t}, t}$ progresses through the updates in Step 8 of the algorithm towards $\Delta_{\hat{y}_{t}, t}$ by growing more positive or more negative depending on the sign of $\Delta_{\hat{y}_{t}, t}$, at an average pace of $\left|\Delta_{\hat{y}_{t}, t}\right|$.

The above behavior is similar to the upper-confidence algorithms under bandit feedback of Auer (2003), Dani et al. (2008) for multiarmed bandits, where our update sign $\beta_{t}$ plays the role there of a random observation whose (conditional) average is the average payoff $\Delta_{\hat{y}_{t}, t}$ of the chosen arm. The randomization in the algorithm serves just to "symmetrize" the unbalanced feedback received in this online protocol, so as to gather information about the true margin $\Delta_{\hat{y}_{t}, t}$ of the chosen class.

We note in passing that the running time of each round of the algorithm includes the time for computing the inversion of the matrix $A_{\hat{y}_{t}, t-1}$, which is $\mathcal{O}\left(d^{2}\right)$ if done incrementally. Calculating $\epsilon_{i, t}$ according to Eq. (9) also takes $\mathcal{O}\left(d^{2}\right)$ for each $i$, computing the projection in Step 2 takes $\mathcal{O}\left(d^{2} K+K \log K\right)$ (see Sect. 3.1). Hence the overall running time per round is $\mathcal{O}\left(d^{2} K+K \log K\right)$. Moreover, it is easy to see that the algorithm can also be run in dual variables (i.e., in a RKHS). This has a twofold implication: (a) The resulting noise model (2) can be made highly nonlinear in the input space, ${ }^{2}$ and (b) the running time per round can be made quadratic in the number of rounds so far, rather than $d^{2}$. In practice, and also in the experiments described in Sect. 5, we actually used a version of the algorithm which maintains a (fully) diagonal matrix $A$ instead of a block-diagonal one. All the steps remain the same except step 8 of Algorithm 1 where we define the $r$ th diagonal element of the matrix to be $\left(A_{t}\right)_{r, r}=\left(A_{t-1}\right)_{r, r}+\left(X_{t}\right)_{r}^{2}$. The running time per round is now $\mathcal{O}(d K+$ $K \log K)$, as all the operations are linear in the dimensions of $X, W$ and $A$, except the projection, as shown next.

\subsection{Computing the projection}

We now show how to compute the projection step efficiently. The running time will be $\mathcal{O}\left(d^{2} K+K \log K\right)$ in the case when $A_{i, t}$ are general matrices, and $\mathcal{O}(d K+K \log K)$ in the case when $A_{i, t}$ are diagonal matrices, where non-diagonal elements equal zero.

Our goal is to solve the following problem, which we write in vector form,

$$
\begin{aligned}
& \arg \min _{\left\{\boldsymbol{u}_{i}\right\}} \frac{1}{2} \sum_{i=1}^{K}\left(\boldsymbol{u}_{i}-\boldsymbol{w}_{i}\right)^{\top} A_{i}\left(\boldsymbol{u}_{i}-\boldsymbol{w}_{i}\right) \\
& \text { s.t. } \quad-\alpha \leq \boldsymbol{u}_{i}^{\top} \boldsymbol{x}, \quad i=1, \ldots, K \\
& \sum_{i=1}^{K} \boldsymbol{u}_{i}^{\top} \boldsymbol{x}=1+\alpha-K \alpha,
\end{aligned}
$$

\footnotetext{
${ }^{2}$ But see also Sect. 4.2 where the linearity assumption is removed at the cost of considering approximation errors to linear noise.
} 
where $A_{i}$ is the $i$-th block of the matrix $A$ and we omitted the example index $t$ for convenience. We change variables for the derivation below and define,

$$
\tilde{\boldsymbol{u}}_{i}=A_{i}^{1 / 2} \boldsymbol{u}_{i}, \quad \tilde{\boldsymbol{w}}_{i}=A_{i}^{1 / 2} \boldsymbol{w}_{i}, \quad \tilde{\boldsymbol{x}}_{i}=A_{i}^{-1 / 2} \boldsymbol{x} .
$$

Substituting back into (6) we get,

$$
\begin{aligned}
& \arg \min _{\left\{\tilde{\boldsymbol{u}}_{i}\right\}} \frac{1}{2} \sum_{i=1}^{K}\left\|\tilde{\boldsymbol{u}}_{i}-\tilde{\boldsymbol{w}}_{i}\right\|^{2} \\
& \text { s.t. } \quad-\alpha \leq \tilde{\boldsymbol{u}}_{i}^{\top} \tilde{\boldsymbol{x}}_{i}, \quad i=1, \ldots, K \\
& \sum_{i=1}^{K} \tilde{\boldsymbol{u}}_{i}^{\top} \tilde{\boldsymbol{x}}_{i}=1+\alpha-K \alpha .
\end{aligned}
$$

Next, we write the Lagrangian and get,

$$
\mathcal{L}=\frac{1}{2} \sum_{i=1}^{K}\left\|\tilde{\boldsymbol{u}}_{i}-\tilde{\boldsymbol{w}}_{i}\right\|^{2}+\sum_{i=1}^{K} \beta_{i}\left(-\alpha-\tilde{\boldsymbol{u}}_{i}^{\top} \tilde{\boldsymbol{x}}_{i}\right)-\lambda\left(\sum_{i=1}^{K} \tilde{\boldsymbol{u}}_{i}^{\top} \tilde{\boldsymbol{x}}_{i}-(1+\alpha-K \alpha)\right) .
$$

Taking the derivative with respect to $\tilde{\boldsymbol{u}}_{i}$ and setting it to zero we get,

$$
\frac{\partial}{\partial \tilde{\boldsymbol{u}}_{i}} \mathcal{L}=\tilde{\boldsymbol{u}}_{i}-\tilde{\boldsymbol{w}}_{i}-\beta_{i} \tilde{\boldsymbol{x}}_{i}-\lambda \tilde{\boldsymbol{x}}_{i}=0
$$

from which we get the optimal solution,

$$
\tilde{\boldsymbol{u}}_{i}=\tilde{\boldsymbol{w}}_{i}+\left(\beta_{i}+\lambda\right) \tilde{\boldsymbol{x}}_{i}
$$

From the KKT conditions we know that $\beta_{i}\left(-\alpha-\tilde{\boldsymbol{u}}_{i}^{\top} \tilde{\boldsymbol{x}}_{i}\right)=0$, thus either $\beta_{i}=0$ or it is chosen such that $-\alpha-\tilde{\boldsymbol{u}}_{i}^{\top} \tilde{\boldsymbol{x}}_{i}=0$, that is $-\alpha=\tilde{\boldsymbol{x}}_{i}^{\top}\left(\tilde{\boldsymbol{w}}_{i}+\left(\beta_{i}+\lambda\right) \tilde{\boldsymbol{x}}_{i}\right)$. We thus get,

$$
\beta_{i}=-\frac{\alpha}{\left\|\tilde{\boldsymbol{x}}_{i}\right\|^{2}}-\frac{\tilde{\boldsymbol{x}}_{i}^{\top} \tilde{\boldsymbol{w}}_{i}}{\left\|\tilde{\boldsymbol{x}}_{i}\right\|^{2}}-\lambda,
$$

where we used the assumption $\|\boldsymbol{x}\|=1$, hence $\left\|\tilde{\boldsymbol{x}}_{i}\right\| \neq 0$ since $A_{i}$ are of full rank. Combining both cases into a single equation we get,

$$
\beta_{i}=\max \left\{0,-\frac{\alpha}{\left\|\tilde{\boldsymbol{x}}_{i}\right\|^{2}}-\frac{\tilde{\boldsymbol{x}}_{i}^{\top} \tilde{\boldsymbol{w}}_{i}}{\left\|\tilde{\boldsymbol{x}}_{i}\right\|^{2}}-\lambda\right\} .
$$

To solve for $\lambda$ we tell apart the set of indices $i$ associated with non-zero values of $\beta_{i}$, formally, $\mathcal{I}=\left\{i: \beta_{i}=0\right\}$. Plugging into the equality constraint we have,

$$
\begin{aligned}
1+\alpha-K \alpha & =\sum_{i} \tilde{\boldsymbol{x}}_{i}^{\top} \tilde{\boldsymbol{u}}_{i} \\
& =\sum_{i} \tilde{\boldsymbol{x}}_{i}^{\top}\left(\tilde{\boldsymbol{w}}_{i}+\left(\beta_{i}+\lambda\right) \tilde{\boldsymbol{x}}_{i}\right) \\
& =\sum_{i} \tilde{\boldsymbol{x}}_{i}^{\top}\left(\tilde{\boldsymbol{w}}_{i}+\left(\max \left\{0,-\frac{\alpha}{\left\|\tilde{\boldsymbol{x}}_{i}\right\|^{2}}-\frac{\tilde{\boldsymbol{x}}_{i}^{\top} \tilde{\boldsymbol{w}}_{i}}{\left\|\tilde{\boldsymbol{x}}_{i}\right\|^{2}}-\lambda\right\}+\lambda\right) \tilde{\boldsymbol{x}}_{i}\right)
\end{aligned}
$$




$$
\begin{aligned}
= & \sum_{i \notin \mathcal{I}} \tilde{\boldsymbol{x}}_{i}^{\top}\left(\tilde{\boldsymbol{w}}_{i}+\left(-\frac{\alpha}{\left\|\tilde{\boldsymbol{x}}_{i}\right\|^{2}}-\frac{\tilde{\boldsymbol{x}}_{i}^{\top} \tilde{\boldsymbol{w}}_{i}}{\left\|\tilde{\boldsymbol{x}}_{i}\right\|^{2}}-\lambda+\lambda\right) \tilde{\boldsymbol{x}}_{i}\right) \\
& +\sum_{i \in \mathcal{I}} \tilde{\boldsymbol{x}}_{i}^{\top}\left(\tilde{\boldsymbol{w}}_{i}+(0+\lambda) \tilde{\boldsymbol{x}}_{i}\right) \\
= & \sum_{i \notin \mathcal{I}} \tilde{\boldsymbol{x}}_{i}^{\top}\left(\tilde{\boldsymbol{w}}_{i}+\left(-\frac{\alpha}{\left\|\tilde{\boldsymbol{x}}_{i}\right\|^{2}}-\frac{\tilde{\boldsymbol{x}}_{i}^{\top} \tilde{\boldsymbol{w}}_{i}}{\left\|\tilde{\boldsymbol{x}}_{i}\right\|^{2}}\right) \tilde{\boldsymbol{x}}_{i}\right)+\sum_{i \in \mathcal{I}} \tilde{\boldsymbol{x}}_{i}^{\top}\left(\tilde{\boldsymbol{w}}_{i}+\lambda \tilde{\boldsymbol{x}}_{i}\right) \\
= & \sum_{i \notin \mathcal{I}}(-\alpha)+\sum_{i \in \mathcal{I}}\left(\tilde{\boldsymbol{x}}_{i}^{\top} \tilde{\boldsymbol{w}}_{i}+\lambda\left\|\tilde{\boldsymbol{x}}_{i}\right\|^{2}\right) \\
= & -\alpha(K-|\mathcal{I}|)+\sum_{i \in \mathcal{I}} \tilde{\boldsymbol{x}}_{i}^{\top} \tilde{\boldsymbol{w}}_{i}+\lambda \sum_{i \in \mathcal{I}}\left\|\tilde{\boldsymbol{x}}_{i}\right\|^{2} .
\end{aligned}
$$

We thus get,

$$
\lambda=\frac{1+\alpha-\alpha|\mathcal{I}|-\sum_{i \in \mathcal{I}} \tilde{\boldsymbol{x}}_{i}^{\top} \tilde{\boldsymbol{w}}_{i}}{\sum_{i \in \mathcal{I}}\left\|\tilde{\boldsymbol{x}}_{i}\right\|^{2}} .
$$

Theoretically, one could enumerate over all possible $2^{K}$ sets $\mathcal{I}$ to solve for $\lambda$ and then $\beta_{i}$ and pick the single consistent solution. The following observation reduces the number of possibilities from $2^{K}$ to $K+1$. Examining (8) we note that $\beta_{i}$ is monotonically decreasing with $\frac{\alpha}{\left\|\tilde{\boldsymbol{x}}_{i}\right\|^{2}}+\frac{\tilde{\boldsymbol{x}}_{\boldsymbol{i}}^{\top} \tilde{\boldsymbol{w}}_{i}}{\left\|\tilde{\boldsymbol{x}}_{i}\right\|^{2}}$. Thus, we have that

$$
\frac{\alpha}{\left\|\tilde{\boldsymbol{x}}_{i}\right\|^{2}}+\frac{\tilde{\boldsymbol{x}}_{i}^{\top} \tilde{\boldsymbol{w}}_{i}}{\left\|\tilde{\boldsymbol{x}}_{i}\right\|^{2}} \geq \frac{\alpha}{\left\|\tilde{\boldsymbol{x}}_{j}\right\|^{2}}+\frac{\tilde{\boldsymbol{x}}_{j}^{\top} \tilde{\boldsymbol{w}}_{j}}{\left\|\tilde{\boldsymbol{x}}_{j}\right\|^{2}} \Rightarrow \beta_{i} \leq \beta_{j} .
$$

Therefore, if $\beta_{j}=0$ (and $j \in \mathcal{I}$ ) then also $\beta_{i}=0$ (and $i \in \mathcal{I}$ ). One last observation is that the set $\mathcal{I}$ cannot be empty. Otherwise, it will imply that $\beta_{i} \neq 0$ for all $i$, and thus $\tilde{\boldsymbol{u}}_{i}^{\top} \tilde{\boldsymbol{x}}_{i}=-\alpha$ for all $i$, in contradiction with the equality constraint $\sum_{i} \tilde{\boldsymbol{u}}_{i}^{\top} \tilde{\boldsymbol{x}}_{i}=1+\alpha-K \alpha$.

All the above yields the following algorithm which we now sketch. First, sort the indices $i$ with respect to a monotonically decreasing order of $\frac{\alpha}{\left\|\tilde{\boldsymbol{x}}_{i}\right\|^{2}}+\frac{\tilde{\boldsymbol{x}}_{i}^{\top} \tilde{\boldsymbol{w}}_{i}}{\left\|\tilde{\boldsymbol{x}}_{i}\right\|^{2}}$. Denote by $r$ the lowest index for which $\beta_{i}=0$. The algorithm starts with $r=1$ and iterates: it computes $\lambda$ and checks if indeed $\alpha_{r}=0$. If yes, the algorithm is done, if not, it increases $r$ and starts over.

Note that we can compute the main quantities directly from the original variables and not the modified ones,

$$
\left\|\tilde{\boldsymbol{x}}_{i}\right\|^{2}=\boldsymbol{x}^{\top} A_{i}^{-1} \boldsymbol{x}, \quad \tilde{\boldsymbol{x}}_{i}^{\top} \tilde{\boldsymbol{w}}_{i}=\boldsymbol{w}_{i}^{\top} \boldsymbol{x} .
$$

These quantities can be precomputed once for all $i$ (this is dominated by the matrix inversions, which takes order of $d^{2} K$ if the inversions are done incrementally) after which we can update the value of $\lambda$ in $\mathcal{O}(1)$. Once $\lambda$ and $\beta_{i}$ have been computed, obtaining the solution to problem (6) can be done via (7) by computing

$$
\boldsymbol{u}_{i}=\boldsymbol{w}_{i}+\left(\beta_{i}+\lambda\right) A_{i}^{-1} \boldsymbol{x}, \quad i=1, \ldots, K
$$

Thus, the total running time of the algorithm is $\mathcal{O}\left(d^{2} K+K \log K\right)$ as claimed. However, in the case when the matrices $A_{i}$ are diagonal, this clearly reduces to $\mathcal{O}(d K+K \log K)$.

Our derivation above is inspired by Crammer and Singer (2002). Other papers where methods have been proposed for solving similar problems include the work of Liu and Ye 
(2009) and of Duchi et al. (2008). Yet, it is not so obvious to us how to adapt their methods to our specific projection problem, while retaining computational efficiency.

\section{Regret analysis}

Our analysis, given in Sect. 7.1, allows us to set the upper confidence level $\epsilon_{i, t}$ in Algorithm 1 in such a way that

$$
\begin{aligned}
\epsilon_{i, t}^{2}= & 2 \boldsymbol{x}_{t}^{\top} A_{i, t-1}^{-1} \boldsymbol{x}_{t}\left(\frac{1}{2}(1+\alpha)^{2}\|U\|_{2}^{2}\right. \\
& \left.+\frac{(1+\alpha)^{2}}{2} \sum_{s=1}^{t-1} \boldsymbol{x}_{s}^{\top} A_{\hat{y}_{s}, s}^{-1} \boldsymbol{x}_{s}+9(1+\alpha)^{2} \log \frac{t+4}{\delta}\right),
\end{aligned}
$$

where $\|U\|_{2}$ is either the actual Euclidean length of the comparison vector $U=\left(\boldsymbol{u}_{1}, \ldots, \boldsymbol{u}_{K}\right)$ defined in (2) or a (known) upper bound thereof. The following is the main theoretical result of this paper, where we emphasize both the data and the time-dependent aspects of the bound.

Theorem 1 In the adversarial setting described so far with $\alpha \in[0,1]$, the cumulative regret $R_{T}$ of Confidit (Algorithm 1) satisfies

$$
R_{T}=O\left(\sqrt{B_{1} T}\left(\sqrt{B_{2} B_{3}}+B_{3}\right)\right)
$$

where

$$
\begin{aligned}
& B_{1}=1+(1+\alpha)^{-2}, \quad B_{2}=\|U\|_{2}^{2}+18 \log ((T+4) / \delta), \\
& B_{3}=\sum_{i=1}^{K} \log \frac{\left|A_{i, T}\right|}{\left|A_{i, 0}\right|} \leq d K \log \left(1+\frac{T}{d K(1+\alpha)^{2}}\right)
\end{aligned}
$$

with probability at least $1-\delta$ uniformly over the time horizon $T$. In the above, $\|U\|_{2}^{2}=$ $\sum_{i=1}^{K}\left\|\boldsymbol{u}_{i}\right\|_{2}^{2}$, and $|\cdot|$ denotes the determinant of the matrix at argument.

The bound described in Theorem 1 is essentially a $\mathcal{O}(\sqrt{T} \log T)$ regret bound, as both $B_{2}$ and $B_{3}$ are logarithmic in $T$. The best previous bound for multiclass prediction in an adversarial bandit setting is $\mathcal{O}\left(T^{2 / 3}\right)$, which was first shown for the Banditron algorithm in the case when also the labels are adversarial, rather than being stochastically generated.

\subsection{Low-noise assumptions}

By making further assumptions on the distribution of the $\boldsymbol{x}_{t}$, such as low noise, one can improve the $\sqrt{T} \log T$ bound and interpolate between $\log ^{2} T$ (hard-margin separation assumption) and $\sqrt{T} \log T$ (no assumptions on $\boldsymbol{x}_{t}$ ). In fact, we can proceed by defining the multiclass margin of a label $y_{t}$ as $\Delta_{b_{t}, t}-\max _{i \neq b_{t}} \Delta_{i, t}$. A low-noise assumption places restrictions (lower bounds) on the probability that $\boldsymbol{x}_{t}$ is drawn in such a way that this margin is small. For instance, under hard-margin separation assumptions we can easily prove the following logarithmic regret result. 
Corollary 1 In the adversarial setting described so far with $\alpha \in[0,1]$, if there is an $\epsilon>0$ such that for any $t$ and any $i \neq b_{t}$ we have $\Delta_{b_{t}, t}-\Delta_{i, t}>\epsilon$, then the cumulative regret $R_{T}$ of Algorithm 1 satisfies

$$
R_{T}=O\left(\frac{B_{1}}{\epsilon}\left(B_{2} B_{3}+B_{3}^{2}\right)\right), \quad \text { for } B_{1}=1+\alpha+\frac{1}{1+\alpha}
$$

where $B_{2}$ and $B_{3}$ are as in Theorem 1.

This result is essentially a $\log ^{2} T$ regret bound, which is a log-factor worse than the one achieved by the random projection-based algorithm described by Kakade et al. (2008), working in the linearly separable case. However, unlike ours, their algorithm does not seem to lend itself to an efficient implementation.

\subsection{General conditional distribution of classes}

In this section we sketch an extension that holds for any conditional distribution of classes $\mathbb{P}(y \mid \boldsymbol{x})$. Because our algorithm in Sect. 3 has been designed to work under a linear noise model, we need to keep track of the approximation error caused by our linear noise hypothesis (2).

Let then $q_{t}$ be the (multiclass) approximation error at time $t$, defined as

$$
q_{t}=q\left(\boldsymbol{x}_{t}\right)=\max _{i=1, \ldots, K}\left|\mathbb{P}\left(y_{t}=i \mid \boldsymbol{x}_{t}\right)-\frac{\alpha+\boldsymbol{u}_{i}^{\top} \boldsymbol{x}_{t}}{\alpha+1}\right|,
$$

i.e., the extent to which instance $\boldsymbol{x}_{t}$ makes the conditional probability of the classes deviate from the linear hypothesis (2). Again, $\boldsymbol{x}_{t}, \boldsymbol{u}_{i}$, and $\alpha$ satisfy the constraints given in Sect. 2, and the cumulative regret is still given by (3), i.e., we are still competing against the best linear hypothesis $b_{t}=b\left(\boldsymbol{x}_{t}\right)=\arg \max _{i=1, \ldots, K}\left(\boldsymbol{u}_{i}^{\top} \boldsymbol{x}_{t}\right)$. Because of the harder setting, we need to modify the upper confidence levels $\epsilon_{i, t}$ so as to incorporate approximation errors. Let Modified Confidit be Algorithm 1 where $\epsilon_{i, t}^{2}$ is given by

$$
\begin{aligned}
\epsilon_{i, t}^{2}= & 2 \boldsymbol{x}_{t}^{\top} A_{i, t-1}^{-1} \boldsymbol{x}_{t}\left(\frac{1}{2}(1+\alpha)^{2}\|U\|_{2}^{2}+\frac{(1+\alpha)^{2}}{2} \sum_{s=1}^{t-1} \boldsymbol{x}_{s}^{\top} A_{\hat{y}_{s}, s}^{-1} \boldsymbol{x}_{s}\right. \\
& \left.+(1+\alpha)^{2} \sum_{s=1}^{t-1} q_{s}+9(1+\alpha)^{2} \log \frac{t+4}{\delta}\right) .
\end{aligned}
$$

We have the following cumulative regret bound.

Theorem 2 In the adversarial setting described above with $\alpha \in[0,1]$, the cumulative regret $R_{T}$ of Modified Confidit satisfies

$$
R_{T}=2 Q_{T}+O\left(\sqrt{B_{1} T}\left(\sqrt{\left(Q_{T}+B_{2}\right) B_{3}}+B_{3}\right)\right),
$$

where

$$
B_{1}=1+(1+\alpha)^{-2}, \quad B_{2}=\|U\|_{2}^{2}+18 \log ((T+4) / \delta),
$$




$$
B_{3}=\sum_{i=1}^{K} \log \frac{\left|A_{i, T}\right|}{\left|A_{i, 0}\right|} \leq d K \log \left(1+\frac{T}{d K(1+\alpha)^{2}}\right)
$$

with probability at least $1-\delta$ uniformly over the time horizon $T$. In the above, $Q_{T}=\sum_{t=1}^{T} q_{t}$ is (an upper bound on) the cumulative approximation error, $\|U\|_{2}^{2}=\sum_{i=1}^{K}\left\|\boldsymbol{u}_{i}\right\|_{2}^{2}$, and $|\cdot|$ denotes the determinant of the matrix at argument.

In order to run Modified Confidit with $\epsilon_{i, t}^{2}$ satisfying (10), we need to know an upper bound on the current approximation error $Q_{t-1}=\sum_{s=1}^{t-1} q_{s}$, the most obvious one being $Q_{t-1}=O(t)$. However, notice that this setting results in useless (i.e., nondecreasing) confidence levels (10), and a vacuous regret bound. Hence Theorem 2 is meaningful only when $Q_{T}$ is sublinear in $T$. Clearly, when $Q_{T}=0$ we recover the result in Theorem 1 .

\section{Experimental study}

We evaluate our algorithm on two domains: text categorization and vowel recognition. In text categorization, we use five natural language classification tasks over nine datasets, with various size and number of labels. In vowel recognition, the goal is to detect spoken vowels as part of the Vocal Joystick project, ${ }^{3}$ again varying in size and number of labels.

Below we summarize the main properties of our datasets. More information can be found in the recent works by Dredze et al. (2008), Crammer et al. (2009a), Lin et al. (2009), where these datasets have previously been used.

\subsection{Data}

The characteristics of the text categorization datasets are summarized in Table 1. We use two datasets based on Amazon product reviews studied previously (Blitzer et al. 2007) for

Table 1 A summary of the nine text categorization datasets, including the number of instances, features and labels and whether the number of examples in each class are balanced

\begin{tabular}{lcccc}
\hline Task & Instances & Features & Labels & Balanced \\
\hline 20 newsgroups & 18,828 & 252,115 & 20 & $\mathrm{Y}$ \\
Amazon7 & 13,580 & 686,724 & 7 & $\mathrm{Y}$ \\
Amazon3 & 7,000 & 494,481 & 3 & $\mathrm{Y}$ \\
Enron A & 3,000 & 13,559 & 10 & $\mathrm{~N}$ \\
Enron B & 3,000 & 18,065 & 10 & $\mathrm{~N}$ \\
NYTD & 10,000 & 108,671 & 26 & $\mathrm{~N}$ \\
NYTO & 10,000 & 108,671 & 34 & $\mathrm{~N}$ \\
NYTS & 10,000 & 114,316 & 20 & $\mathrm{~N}$ \\
Reuters & 685,071 & 268,170 & 4 & $\mathrm{~N}$ \\
\hline
\end{tabular}

${ }^{3}$ http://ssli.washington.edu/vj. 
domain classification. The first dataset Amazon7 contains all seven product types: books, dvds, music, apparel, electronics, kitchen and, video. The second smaller subset includes reviews from only the first three product types: books, dvds, and music. Preprocessing and feature generation followed the protocol of Blitzer et al. (2007).

The 20 newsgroups $^{4}$ is a very popular dataset with about 20,000 newsgroup messages, divided into 20 different newsgroups. This dataset was also used in the past for evaluation both in the supervised binary and multi-class text classification setting, as well as in the unsupervised clustering setting. Each message was represented as a binary bag-of-words.

Two additional datasets are based on the automatic classification of Enron emails into one of the 10 largest folders. ${ }^{5}$ Two users were selected: farmer-d (Enron A) and kaminski-v (Enron B). The classes were defined using the ten largest folders for each user (excluding non-archival email folders such as "inbox," "deleted items," and "discussion threads"). Emails were represented as binary bag-of-words with stop-words removed.

The NY Times (Sandhaus 2008) dataset was published few years ago. The entire dataset contains 1.8 million articles, published across 20 years starting from 1987. This is an ideal corpus for large scale NLP tasks, as it is one of the largest publicly released annotated news text. We used a subset with three annotations for each article: the desk that produced the story (Financial, Sports, etc.) (NYTD), the online section to which the article was posted (NYTO), and the section in which the article was printed (NYTS). Articles were represented as bag-of-words with feature counts (stop-words removed).

Finally, we also used documents from RCV1v2 dataset (Lewis et al. 2004), which contains more than 800,000 manually categorized newswire stories (RCV1-v2/ LYRL2004). Each article contains one or more labels describing its general topic, industry, and region. We performed topic classification with the four general topics: corporate, economic, government, and markets.

In all of the above text categorization datasets, we followed the experimental setting described by Crammer et al. (2009a), including data preprocessing.

The vowel recognition datasets are taken from the Vocal Joystick Vowel Corpus (Kilanski et al. 2006) collected specifically for the VJ project. ${ }^{6}$ We followed the same experimental setting of Lin et al. (2009) which we repeat now for completeness. We used a training set built from 21 recording sessions ( 2 speakers appear twice, although there is only partial overlap in their sounds), a development set of 4 speakers, and a test set of 10 speakers. All speakers come from the earlier data collection efforts described by Kilanski et al. (2006) and capture the wide variability in human vowel production.

We tested two sizes of label sets: 4-vowel (æ, a, u, i) classification and 8-vowel (with additional four vowels: a, o, i, e) classification. For the 4 -vowel case, there are about $275 \mathrm{~K}$ training examples or frames (1,931 utterances), for the 8-vowel case there are about $550 \mathrm{~K}$ frames $(3,867$ utterances). The test set has $116 \mathrm{~K}$ examples or frames (716 utterances) for 4-vowel task and $236 \mathrm{~K}$ (1432 utterances) frames for 8-vowel task. In these tasks, each utterance contains a single speaker uttering a single vowel. We used MFCCs with first-order deltas yielding vectors with 26 distinct features (frames were $25 \mathrm{~ms}$ long with a $10 \mathrm{~ms}$ shift) and we also varied the number of frames in the feature window. There are 53 features when using one frame and 365 features when using seven frames. The above gave rise to four vowel recognition datasets, named v4.w1 (four vowels, one frame), v4.w7 (four vowels, seven frames), v8.w1 (eight vowels, one frame), and v8.w7 (eight vowels, seven frames).

\footnotetext{
${ }^{4}$ http://people.csail.mit.edu/jrennie/20Newsgroups/.

${ }^{5}$ http://www.cs.cmu.edu/ enron/.

${ }^{6}$ The VJ corpus is freely available online at http://ssli.washington.edu/vj.
} 
Table 2 Tested versions of Confidit

\begin{tabular}{lllll}
\hline Version & Projection & Upper conf. $\epsilon_{i, t}^{2}$ & Covariance & Comment \\
\hline 1 & yes & $(9)$ & Full & Algorithm 1 \\
2 & no & $(11)$ & Full & \\
3 & no & $(9)$ & Full & \\
4 & yes & $(11)$ & Full & \\
5 & no & $(11)$ & Diagonal & Used in subsequent \\
& & & & experiments \\
\hline
\end{tabular}

\subsection{Algorithms}

We evaluated five algorithms: two of them work in the bandit setting, the other three in the full information setting. The two bandit algorithms are the Banditron algorithm (Kakade et al. 2008), and the following modification to our algorithm. First, as is typical of many upper confidence-based algorithms, the width of the confidence interval is a pessimistic overestimation of the actual uncertainty, which suggests that implementing our algorithm and testing it on real data in the exact form given by the theory may not work well in practice. Hence, we replaced the multiplier of $\boldsymbol{x}_{t}^{\top} A_{i, t-1}^{-1} \boldsymbol{x}_{t}$ in the definition of $\epsilon_{i, t}^{2}$ (see Eq. (9)) with some constant $\eta$ whose value was set by cross validation, that is, we used ${ }^{7}$

$$
\epsilon_{i, t}^{2}=\eta \boldsymbol{x}_{t}^{\top} A_{i, t-1}^{-1} \boldsymbol{x}_{t} .
$$

Second, the projection step in Algorithm 1 is only needed for technical purposes in the analysis (operating with a bounded martingale difference sequence). On preliminary experiments (not reported here) we observed that the actual length of the vectors either did not grow large or, when it did, the projection actually hurted performance-see below. Furthermore, the real-world data do not necessarily satisfy the constraint of Eq. (5). Thus, we decided to remove this projection step from our implementation of Algorithm 1. Third, for computational efficiency reasons, the inverse matrices $A_{i, t-1}^{-1}$ have been replaced by diagonal versions thereof.

In order to illustrate the properties of the three abovementioned implementation choices for Confidit (full vs. diagonal matrices, projection vs. no projection, theoretical vs. simplified confidence intervals), and to motivate our subsequent setting on the real-world data, we run a simple simulated experiment on synthetic data. Our goal is to underscore the contribution of each to the final performance. We ran five versions of Confidit, ranging from "theoretical" to "practical". These are summarized in Table 2.

We repeated the following experiment 10 times, the reported results being an average over the 10 runs. On each run we generated 1,000 random examples of dimension 9, where the first 5 features were used to generate labels, and the remaining 4 features are random with zero mean and unit standard deviation, with no correlation with other features or labels. One of five labels (this is a multiclass problem with $K=5$ ) were generated using five (random) linear models $\boldsymbol{u}_{i}$ of dimension five through the first 5 features. Specifically, a given instance vector $\boldsymbol{x}=\left(x_{1}, \ldots, x_{9}\right)$ is associated with class $i=\arg _{\max } \operatorname{mat}_{j=1, \ldots, 5}\left(\boldsymbol{u}_{j}, 0,0,0,0\right)^{\top} \boldsymbol{x}$. The norm of the models $\boldsymbol{u}_{i}$ was recorded, since Eq. (9) depends on it. Notice that these data

\footnotetext{
${ }^{7}$ On top of this, observe that the multiplier in (9) is dependent on the norm $\|U\|$ of the comparison classifier, which need not be known to the algorithm.
} 
Fig. 2 Averaged (over ten random generations of data) cumulative number of mistakes for each of the five variants and seven choices of parameters

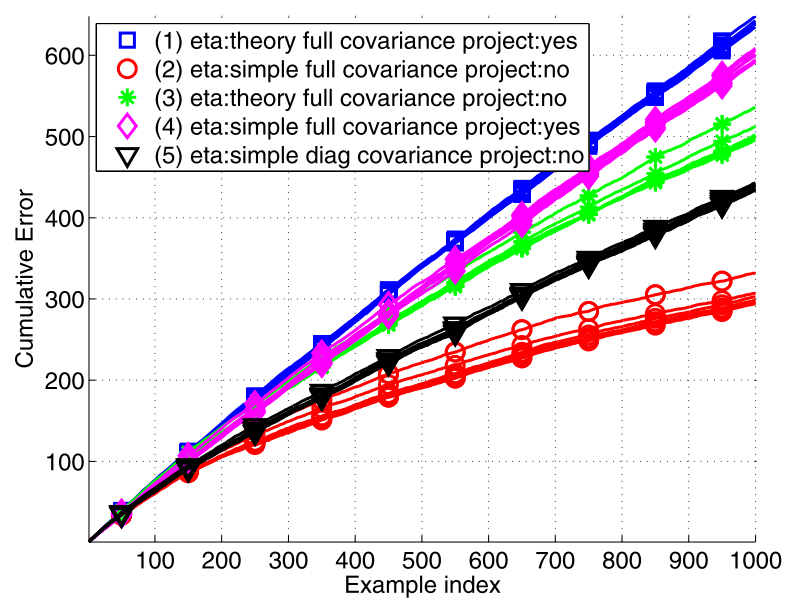

are not generated according to model (2), thereby making the setting of $\alpha$ in Confidit (and the associated projection step) lose much of its significance. In fact, in all cases we set $\alpha=1$, turning Confidit into a deterministic algorithm.

We ran each of the five versions seven times. When (9) was used (variants 1 and 3) we set $\|U\|$ to be the squared norm of the model used to generated the data, scaled by one of the 7 values $10^{-3}, 10^{-2}, \ldots, 10^{3}$, which were meant to provide a further "knob" to Eq. (9), possibly capturing the variability due to the confidence level $\delta$ therein. Otherwise, when (11) was used (variants 2,4 and 5), we set $\eta=10^{-3}, 10^{-2}, \ldots, 10^{3}$, seven values all together.

The results are summarized in Fig. 2 showing the (average) cumulative number of mistakes for each of the five variants and the seven choices of parameters. Interestingly, Algorithm 1 (shown in blue squares) performs worst, with more than 600 mistakes after 1,000 examples. The version that uses (11) rather than (9) (magenta diamonds) performs slightly better, with slightly less than 600 mistakes. The versions with no projection perform better, where the one that uses (9) (green stars) makes about 500 mistakes and the one that uses (11) (red circles) performing best, with about 300 mistakes. If we use diagonal matrices rather than full ones (black triangles), the performance drops and the number of mistakes increases to about 400 . This fifth version is the one we used in our subsequent experiments, since it is much faster than all other (full matrix) versions.

In summary, these simple findings suggest that indeed the projection step in Confidit is only needed for technical purposes related to the specifics of our noise model, and that the confidence intervals computed by theory in (9) are too pessimistic. It seems that the full matrix version performs best, yet it is unfortunate that in many real-world problems it is not feasible to manipulate such matrices. It is also important to observe that performance is robust to the specific choice of parameters, as in most variants the total number of mistakes is within a range of about 30 .

The three Confidit's competitors that work in the full information setting are the classical Perceptron algorithm (Rosenblatt 1958) extended to the multiclass setting as by Crammer and Singer (2003), a diagonal multiclass version of the 2nd-order perceptron algorithm (Cesa-Bianchi et al. 2005), and AROW (Crammer et al. 2009b). Note that only AROW is margin-based among all five algorithms. All other algorithms are mistake driven (at most). This difference will be reflected in the performance of the algorithms.

In all experiments with real-world data we performed 10-fold cross validation for all algorithms. Algorithm's parameters ( $\gamma$ for Banditron, $r$ for AROW, $\eta$ for Confidit and $a$ 
for the 2 nd order Perceptron) were tuned using a single split of the data into $80 \%$ training and $20 \%$ evaluation. In a preliminary set of experiments we also evaluated the influence of the parameter $\alpha$ for Confidit. Since the optimal value was very close to 1 anyway, with no significant improvement in the results, we decided to $\operatorname{set}^{8} \alpha=1$ even on the real-world datasets.

\section{Empirical results}

We now report our results on real-world datasets, starting from text categorization. We summarize these results both in terms of cumulative number of mistakes in the online setting and in terms of test error in the batch setting, beginning with the former.

\subsection{Online results for text categorization}

Evaluation of all algorithms in the online setting is split into Figs. 3 and 4. We refer to the best scoring label (prediction) as $\tilde{y}_{t}=\arg \max _{i} \boldsymbol{w}_{i, t-1}^{\top} \boldsymbol{x}_{t}$, and to the one actually output as $\hat{y}_{t}$. For Perceptron, 2nd-order Perceptron and AROW, these two are always the same. On the contrary, bandit algorithms work in the partial information setting, hence their need to make prediction $\tilde{y}_{t}$ and output label $\hat{y}_{t}$ be generally different. In particular, Banditron outputs a uniformly random label with some fixed probability, while Confidit outputs the label with the highest sum of score and confidence $\boldsymbol{w}_{i, t-1}^{\top} \boldsymbol{x}_{t}+\epsilon_{i, t}$.

We refer now to Fig. 3 which summarizes the results for four representative datasets. Similar observations hold for the other five datasets shown in Fig. 4. The left column of Fig. 3 summarizes the cumulative number of prediction mistakes each of the five online algorithms makes during its first training epoch over the data. The plots are in a linear-linear scale. The four datasets are (top to bottom): 20 newsgroups, Amazon3, NYTS, and Reuters. We observe that AROW makes the least number of mistakes, while Banditron makes the most. There is no clear ordering between the other algorithms. This is surprising since Confidit has only partial information, on each iteration, while Perceptron and 2nd-order Perceptron do rely on full label information. Furthermore, we expected algorithms that employ (diagonal) 2nd-order information (e.g., 2nd-order Perceptron), to outperform algorithms that are based only on first order information (e.g., Perceptron).

The second column of Fig. 3 summarizes the cumulative number of prediction mistakes over ten training epochs over the data. The plots are in a log-log scale. The vertical dashed black line indicates the end of the first epoch. In general, we observe a trend similar to the one observed after the first epoch. Banditron makes the same rate of mistakes in later epochs as the first one, yet it is not always the case for the other algorithms. For example, on 20 newsgroups (top plots) all other algorithms make noticeably fewer mistakes in the last 9 training epochs (on average) compared to the number of mistakes they make in the first iteration. The parameters of all algorithms were the ones that minimize the error on held-out-data, which may be far from optimal when evaluating just on the number of online mistakes.

Finally, the right column of Fig. 3 summarizes the cumulative number of examples for which the prediction and the output of bandit-based algorithms are not the same. This may

\footnotetext{
${ }^{8}$ Recall that $\alpha$ quantifies our prior knowledge about the amount of overlap among the $K$ classes. This prior knowledge is enforced through the projection step in Confidit. Since we decided to remove the projection from our implementation, the role of $\alpha$ indeed loses much of its practical significance.
} 

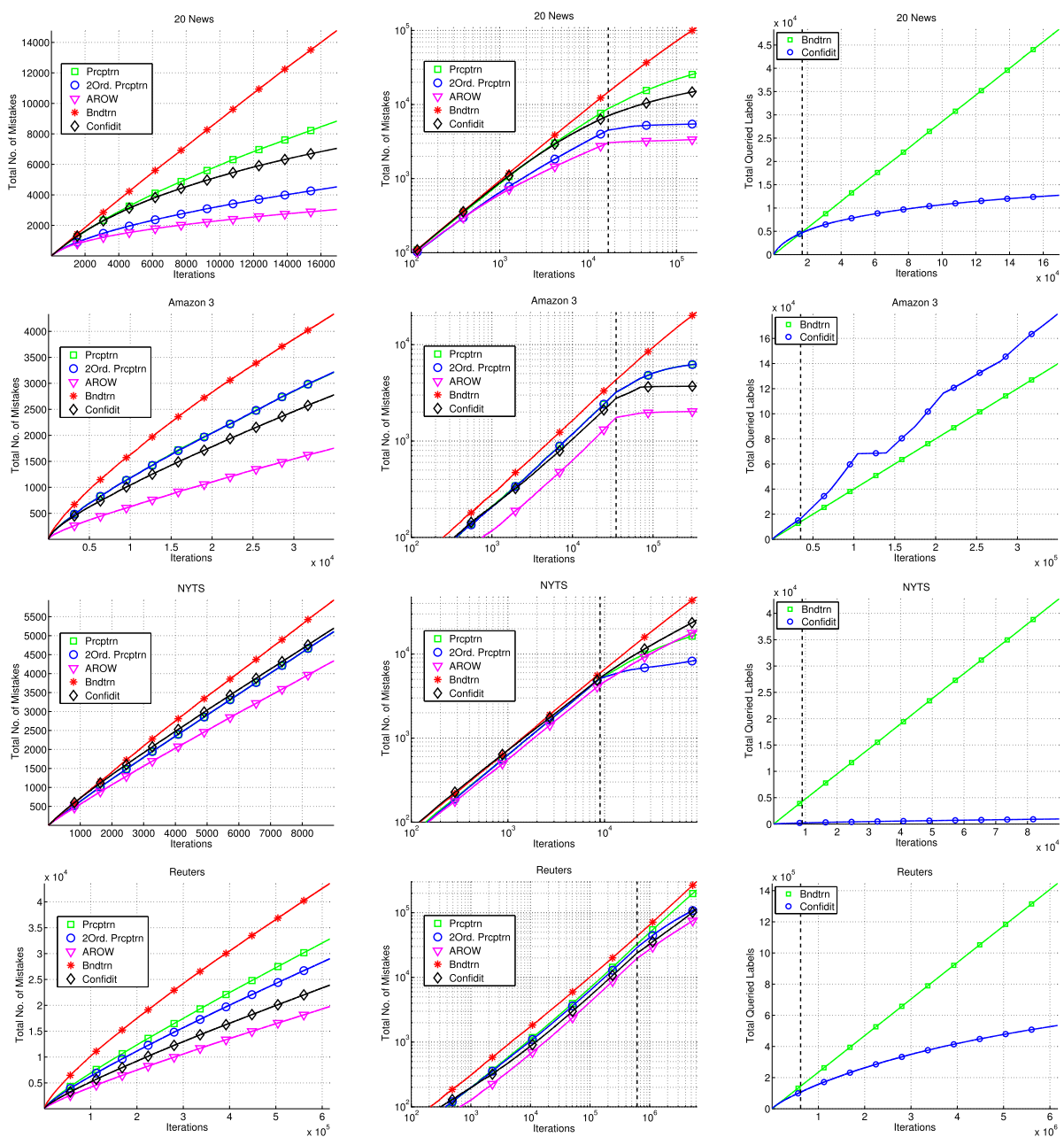

Fig. 3 Cumulative number of prediction mistakes in the first training epoch (left; linear-linear scale), and in all 10 epochs (middle; log-log scale), no label noise. The right plots shows the cumulative number of examples for which a prediction $\hat{y}$ of a bandit algorithm is not the label output $\tilde{y}$. Four datasets are used (top to bottom): 20 newsgroups, Amazon3, NYTS and Reuters

be thought of as an exploration rate or total queried labels-which is the total number of time for which the algorithm output a label which is not the best according to the model. Clearly, this only applies to Banditron and Confidit. The plots are in linear-linear scale. By definition, Banditron has a fixed exploration rate (of $\gamma$ ), as opposed to Confidit whose exploration rate varies as more examples are observed. In seven out of the nine datasets (four are plotted in Fig. 3, the other five are in Fig. 4) Confidit has lower and monotonically decreasing exploration rate. Confidit has more cumulative exploration rate in two of the datasets: Amazon3 and Amazon7. A possible explanation is that the number of features in these datasets is large (the largest among the nine datasets). Since the exploration rate of Confidit is based on a term which is linear in the square of the features, and there are many rare features, new features are introduced at a high-rate during training. As a result, 

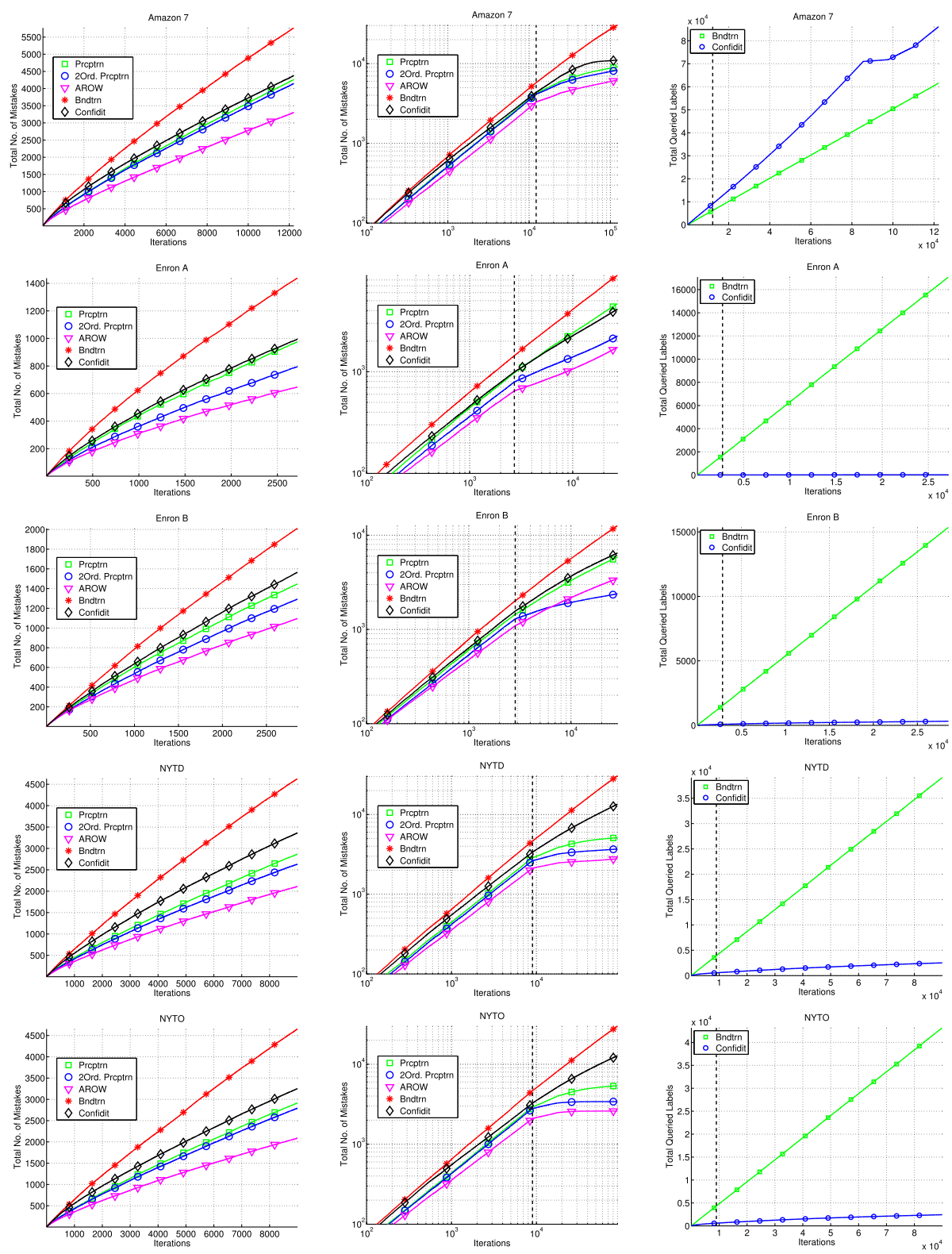

Fig. 4 Same as Fig. 3, with the remaining five datasets (top to bottom): Amazon7, Enron A, Enron B, NYTD, and NYDO

Confidit gets biased towards exploring classes with a high number of relatively rare features.

\subsection{Batch evaluation for text categorization}

Table 3 summarizes averaged 10-fold cross-validation error rates. Bold entries indicate best results within all algorithms except AROW (which is margin based), while underlined en- 

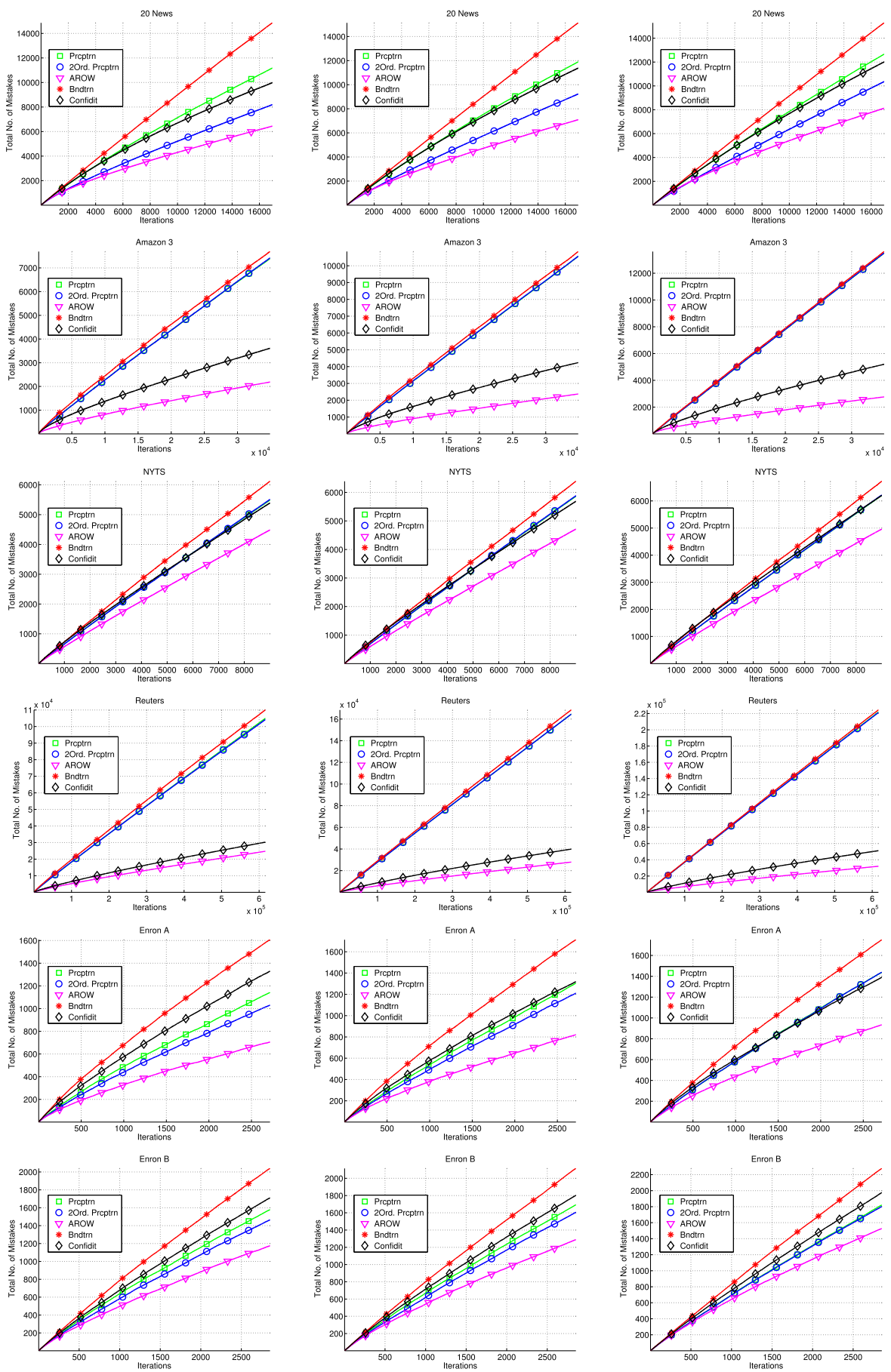

Fig. 5 Cumulative number of prediction mistakes in the first training epoch in linear-linear scale for three noise levels $10 \%, 20 \%$ and $30 \%$ (left to right). Six datasets are shown (top to bottom): 20 newsgroups, Amazon3, NYTS, Reuters, Enron A and Enron B 
Table 3 Test error with 10-fold cross validation with no label noise. Bold entries indicate lowest error-rate among the Perceptron, 2nd order Perceptron, Banditron and Confidit algorithms (AROW is not included, since it is margin-based, unlike the others), and underlined entries indicates lowest error-rate between the two bandit algorithms. Additional $\dagger$ and $\star$ indicate $p$-value of 0.01 and 0.001 respectively, when comparing all results, while $(\dagger)$ and $(\star)$ only include comparison between the two bandit-based algorithms

\begin{tabular}{lccccr}
\hline Task & Perceptron & $\begin{array}{l}\text { Second order } \\
\text { perceptron }\end{array}$ & AROW & Banditron & Confidit \\
\hline 20 newsgroups & 20.04 & 13.92 & 8.14 & 50.52 & $\mathbf{1 2 . 4 1 \star}$ \\
Amazon7 & 25.71 & 25.73 & 22.30 & 29.15 & $\mathbf{2 4 . 7 3}(\star)$ \\
Amazon3 & 5.52 & $\mathbf{5 . 4 5}$ & 4.47 & 7.54 & $6.67(\star)$ \\
Enron A & 21.39 & $\mathbf{1 9 . 5 7}$ & 16.39 & 27.35 & $23.01(\star)$ \\
Enron B & 34.02 & $\mathbf{3 3 . 3 2}$ & 27.11 & 42.49 & $37.89(\star)$ \\
NYTD & 22.02 & $\mathbf{2 0 . 9 5}$ & 17.61 & 31.23 & $27.02(\star)$ \\
NYTO & $\mathbf{2 1 . 8 4}$ & 22.69 & 17.20 & 31.64 & $25.20(\star)$ \\
NYTS & $\mathbf{4 9 . 1 6}$ & 51.23 & 43.05 & 56.06 & $54.24(\dagger)$ \\
Reuters & 4.29 & 3.96 & 2.92 & 4.59 & $\mathbf{3 . 5 5}$ \\
\hline
\end{tabular}

tries indicate superiority when comparing only the two bandit-based algorithms Banditron and Confidit. It is not surprising that AROW outperforms all other algorithms, as it works in the full information case with a margin-sensitive update rule. (This was shown for the binary case by Crammer et al. (2009b)). Thus we consider below only Perceptron and 2nd-order Perceptron in the full information setting, and their bandit counterparts: Banditron and Confidit. When comparing the two algorithms working in the full information setting, we see that 2nd-order Perceptron outperforms Perceptron in 6 datasets, and is worse in 2 (there is a tie on one dataset). In fact the 2nd-order Perceptron outperforms all other three algorithms in 4 datasets. Confidit performs best among all four algorithms (including full information ones) in three datasets, and outperforms Banditron in all dataset. All results, except one, are statistically significant with $p$-value of 0.001 .

\subsection{Label noise for text categorization}

We repeated the above experiments with artificial label noise injected into the training data. Specifically, we picked examples from the training set with probability $p$, and replaced the true label of these examples with a uniformly distributed random label. This process was performed only for the training subset of the data, the test results were evaluated using uncorrupted data. However, parameter tuning was performed using only corrupted data. The motivation here is that injecting label noise might be more harmful in the bandit setting than the full information setting. This is because the bandit algorithms not only observe partial information, but when they get some, this information may be incorrect.

Online results for six datasets are given in three groups of plots. Figure 5 shows the cumulative number of mistakes (compared to the true non-corrupted label) for three noise levels: $10 \%, 20 \%$, and $30 \%$ (left to right), similar to the left column of Fig. 3 . Figure 6 shows the cumulative number of mistakes (compared to the true non-corrupted label) on 10 training epochs for the three noise levels: $0 \%$ (no noise), ${ }^{9} 20 \%$, and $30 \%$ (left to right), to

\footnotetext{
${ }^{9}$ The left column Fig. 6 shows the results for $0 \%$ label noise, rather than $10 \%$, as in Fig. 5. This is because, after the first iteration the performance of most algorithms on noisy data has similar characteristics, as
} 

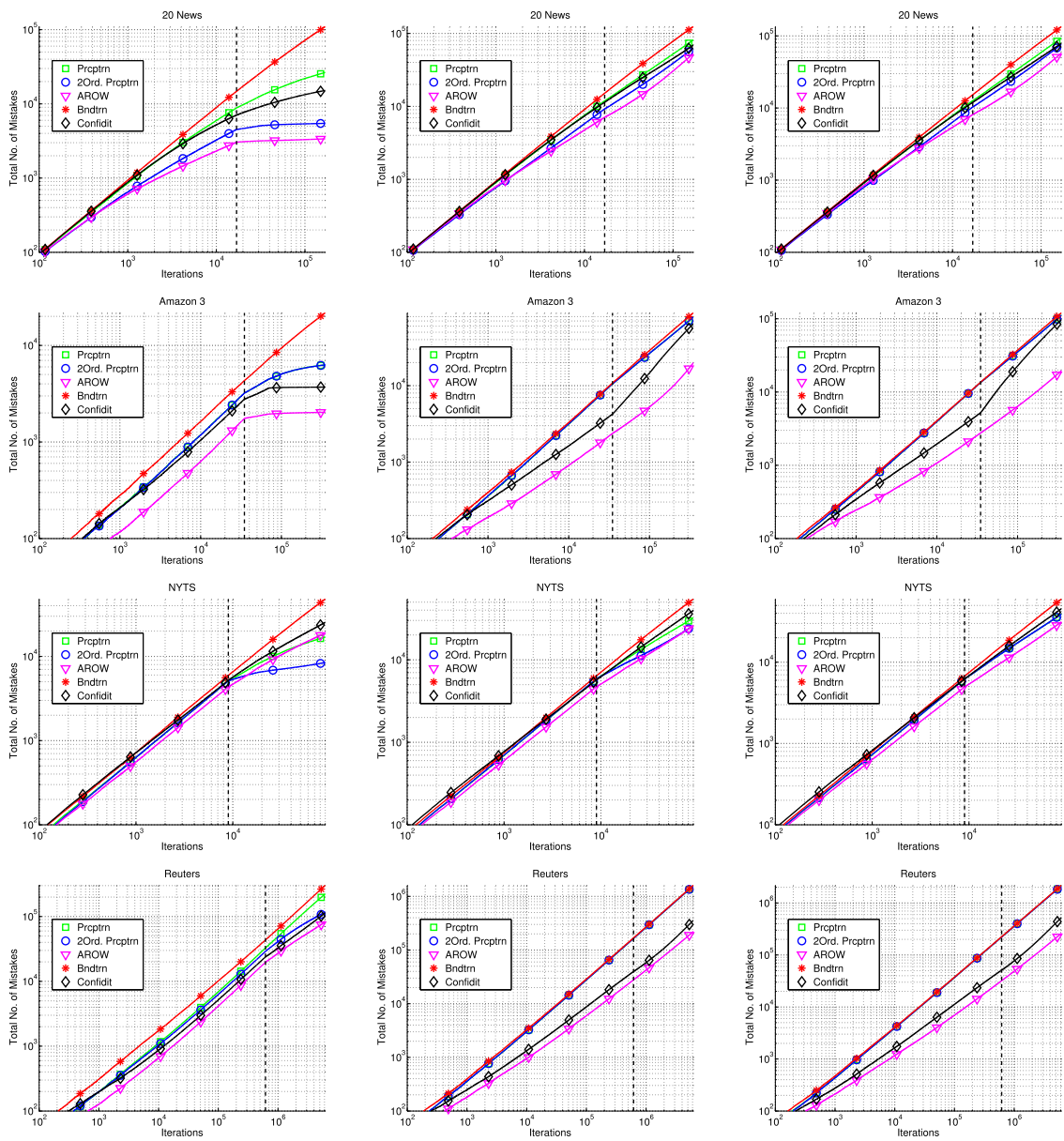

Reuters
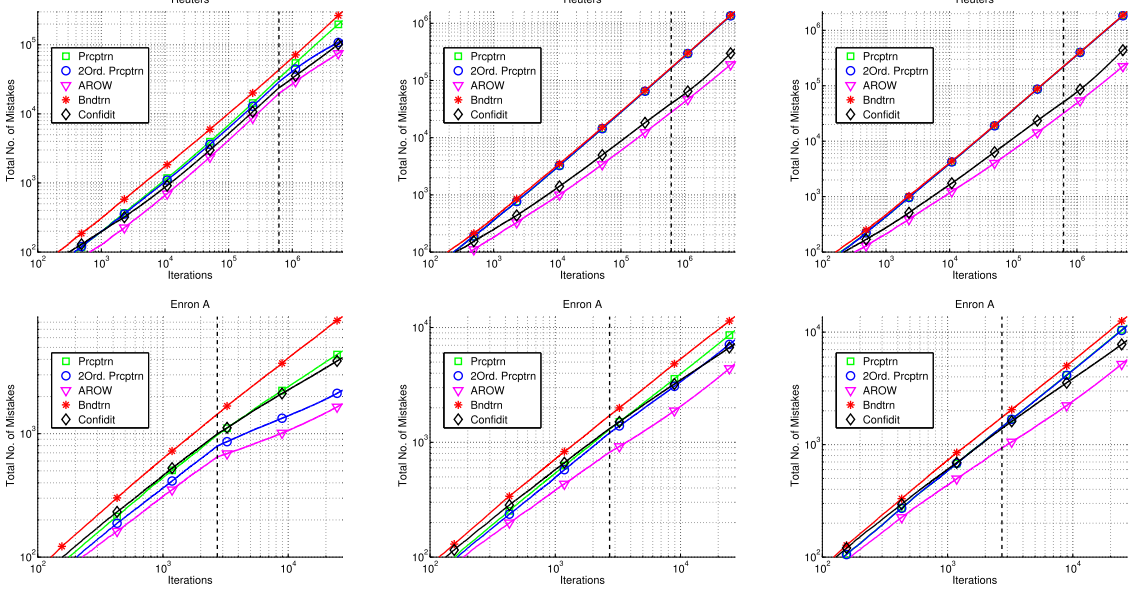

Enron A

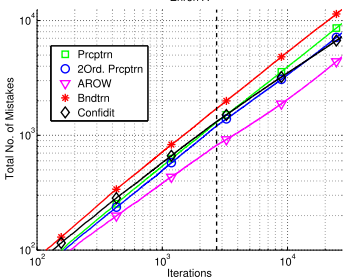

Enron A
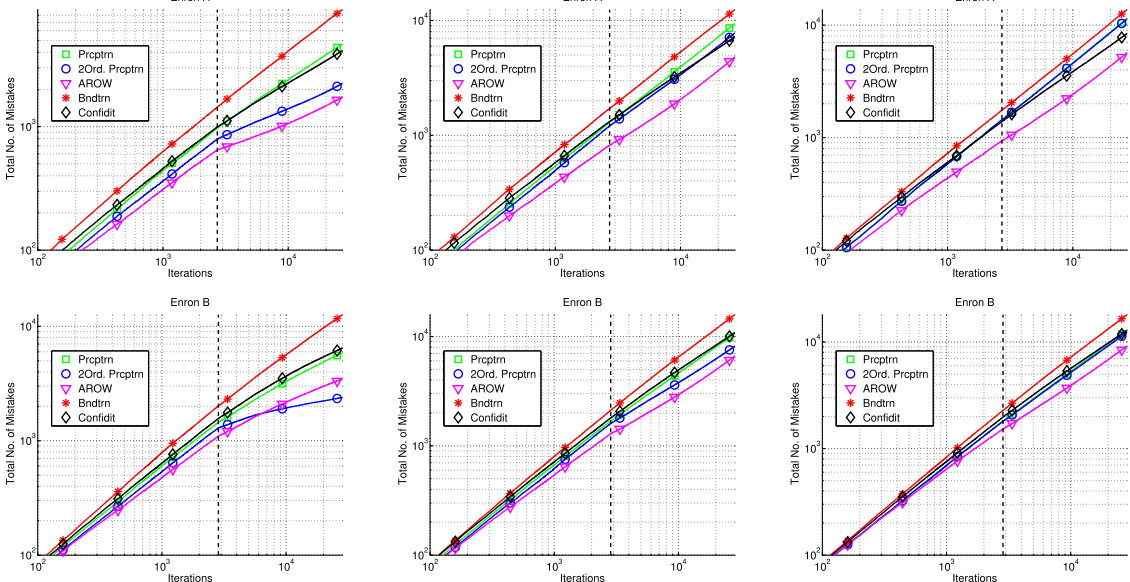

Fig. 6 Cumulative number of prediction mistakes in 10 training epochs in linear-linear scale for three noise levels $0 \%, 20 \%$ and $30 \%$ (left to right). Six datasets are shown (top to bottom): 20 newsgroups, Amazon3, NYTS, Reuters, Enron A and Enron B 
Table 4 Same as Table 3, with $10 \%$ training label noise

\begin{tabular}{lllllr}
\hline Task & Perceptron & $\begin{array}{l}\text { Second order } \\
\text { perceptron }\end{array}$ & AROW & Banditron & Confidit \\
\hline 20 newsgroups & 31.27 & 22.03 & 13.10 & 53.04 & $\mathbf{1 7 . 7 0 \star}$ \\
Amazon7 & 30.96 & 31.53 & 23.46 & 34.74 & $\mathbf{2 9 . 4 9 \dagger}$ \\
Amazon3 & 12.51 & 12.17 & 5.42 & 16.12 & $\mathbf{8 . 9 0 \star}$ \\
Enron A & 32.52 & 27.65 & 19.73 & 36.85 & $\mathbf{2 7 . 5 8}(\star)$ \\
Enron B & 41.14 & $\mathbf{4 0 . 6 4}$ & 34.05 & 46.15 & $41.27(\star)$ \\
NYTD & 28.46 & $\mathbf{2 8 . 1 5}$ & 19.57 & 37.60 & $28.94(\star)$ \\
NYTO & 29.35 & $\mathbf{2 7 . 6 6}$ & 20.14 & 39.00 & $27.87(\star)$ \\
NYTS & $\mathbf{5 3 . 3 1}$ & 54.55 & 44.26 & 58.22 & $54.82(\star)$ \\
Reuters & 16.79 & 16.86 & 3.64 & 18.92 & $\mathbf{5 . 9 6}$ \\
\hline
\end{tabular}

be compared to the middle column of Fig. 3. Finally, Fig. 7 shows the cumulative number of examples for which the prediction and the output of bandit-based algorithms are not the same for the three noise levels: $10 \%, 20 \%$ and $30 \%$ (left to right), similar to the right column of Fig. 3. As before, this may be thought of as an exploration rate, and it only applies to Banditron and Confidit.

Comparing the three columns of Fig. 5 per row, we observe that all algorithms make more mistakes, still the relative ordering remains the same. For example, the 2nd-order Perceptron algorithm makes about 8,000 mistakes on the 20 newsgroups data set of which $10 \%$ of the labels were corrupted, about 9,000 mistakes when $20 \%$ of the labels were corrupted, and about 10,000 mistakes when $30 \%$ of the labels were corrupted. Similar trend is observed for Confidit which makes about 10,000, 11,500 and 12,000 mistakes when run on the same data corrupted with $10 \%, 20 \%$ and $30 \%$ label-noise, respectively.

Comparing the three columns of Fig. 6 per row, we observe that, when run on noisy data (second and third columns), all algorithms continue to make mistakes after the first epoch at a rate that remains similar throughout time. This is in contrast to the evidence we collected on noiseless data (left column), where the mistake rate curves tend to flatten out in later epochs. The phenomenon was observed in the full information setting as well: Since the data are not separable the algorithms will continue to make mistakes.

Additionally, from Fig. 7 we see that compared to the noise-free case, Confidit has more exploration in some datasets, and comparable in others. In these plots we show the total queried labels - the total number of time for which the algorithm output a label which is not the best according to the model. This is also due to the different value of explorationexploitation parameter $\eta$ automatically set by cross-validation. For example, in the first row of Fig. 7 we see that for 20 newsgroup Confidit has about 20,000 queries altogether for $10 \%$ label noise, about 25,000 queries for $20 \%$ label noise, and about 40,000 queries for $30 \%$ label noise. This relation can also be observed on the EnronB dataset (last row). On other datasets, the trend is inverse (less queries with more noise, e.g., EnronA, 2nd line form the bottom), and on others there is no clear trend.

Finally, averaged 10-fold cross validation error rates on label noise rates $10 \% 20 \%$, and $30 \%$ are summarized in Tables 4, 5 and 6, respectively. Evaluations are performed using uncorrupted data, the noisy data being used only during training and parameter tuning. There

opposed to non-noisy data. However, on the first epoch the characteristics are changing gradually when we increase the label noise. 

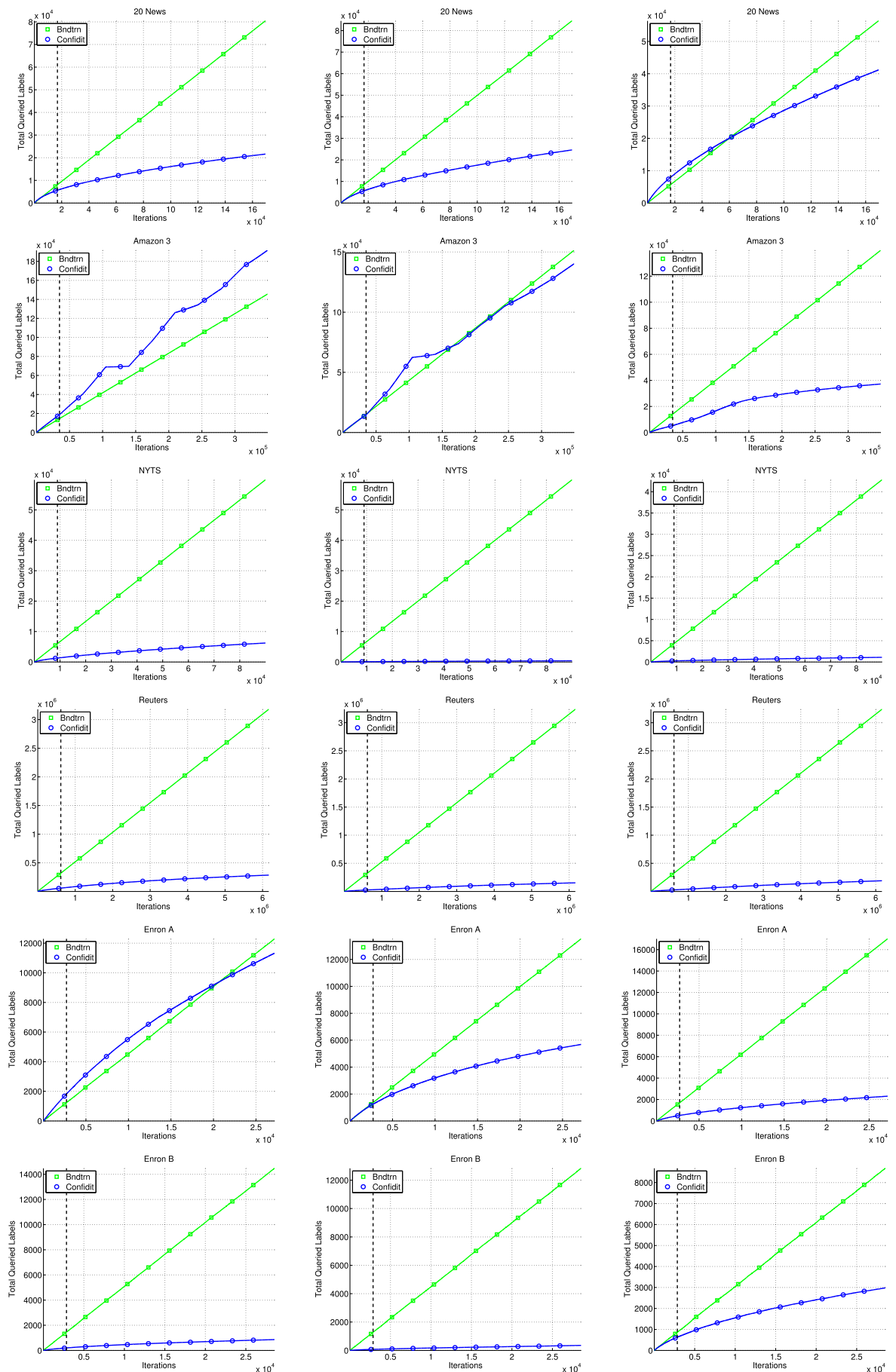

Fig. 7 The cumulative number of examples for which a prediction $\hat{y}$ of a bandit algorithm is not the label output $\tilde{y}$ for three noise levels $10 \%, 20 \%$ and $30 \%$ (left to right). Six datasets are shown (top to bottom): 20 newsgroups, Amazon3, NYTS, Reuters, Enron A and Enron B 
Table 5 Same as Table 3, with $20 \%$ training label noise

\begin{tabular}{lllccc}
\hline Task & Perceptron & $\begin{array}{l}\text { Second order } \\
\text { perceptron }\end{array}$ & AROW & Banditron & Confidit \\
\hline 20 newsgroups & 38.33 & 30.54 & 18.49 & 67.38 & $\mathbf{2 3 . 6 7 \star}$ \\
Amazon7 & 35.49 & 41.63 & 25.02 & 39.46 & $\mathbf{3 2 . 8 0 \star}$ \\
Amazon3 & 20.04 & 19.34 & 6.94 & 23.80 & $\mathbf{1 1 . 2 9 \star}$ \\
Enron A & 38.77 & 36.62 & 22.75 & 43.15 & $\mathbf{2 9 . 4 0 \star}$ \\
Enron B & 50.48 & 46.50 & 36.63 & 54.89 & $\mathbf{4 5 . 1 4 \star}$ \\
NYTD & 36.90 & 34.92 & 21.46 & 43.99 & $\mathbf{3 1 . 6 3 \star}$ \\
NYTO & 35.62 & 34.26 & 21.81 & 45.17 & $\mathbf{3 1 . 1 6}$ \\
NYTS & 59.42 & 57.69 & 46.82 & 61.77 & $\mathbf{5 4 . 9 0 \star}$ \\
Reuters & 25.94 & 26.46 & 4.03 & 28.77 & $\mathbf{8 . 5 8}$ \\
\hline
\end{tabular}

Table 6 Same as Table 3, with $30 \%$ training label noise

\begin{tabular}{llllll}
\hline Task & Perceptron & $\begin{array}{l}\text { Second order } \\
\text { perceptron }\end{array}$ & AROW & Banditron & Confidit \\
\hline 20 newsgroups & 45.88 & 39.92 & 21.41 & 65.71 & $\mathbf{3 0 . 4 5 \star}$ \\
Amazon7 & 42.33 & 41.41 & 27.47 & 45.28 & $\mathbf{3 6 . 2 4} \star$ \\
Amazon3 & 28.70 & 27.38 & 6.22 & 31.70 & $\mathbf{1 7 . 1 6 \star}$ \\
Enron A & 46.85 & 46.09 & 24.04 & 49.20 & $\mathbf{3 3 . 0 1 \star}$ \\
Enron B & 55.52 & 54.89 & 37.17 & 61.10 & $\mathbf{5 0 . 5 7 \star}$ \\
NYTD & 43.14 & 43.36 & 24.40 & 50.74 & $\mathbf{3 4 . 9 3 \star}$ \\
NYTO & 44.43 & 42.40 & 24.78 & 48.85 & $\mathbf{3 3 . 8 5 \star}$ \\
NYTS & 63.39 & 63.66 & 48.99 & 65.63 & $\mathbf{5 5 . 8 4 \star}$ \\
Reuters & 35.34 & 35.70 & 4.53 & 36.61 & $\mathbf{1 1 . 7 0 \star}$ \\
\hline
\end{tabular}

are few trends. First, as expected, performance degrades as the level of noise increases. For example, on the Amazon7 dataset the error rate of Banditron increases from $29.15 \%$ via $34.74 \%$ via $39.46 \%$ to $45.28 \%$ as the label noise levels range from $0 \%$ (see Table 3 ) to $10 \%$ to $20 \%$ to $30 \%$. Second, as the level of noise increases, it seems that Confidit suffers the least compared to Perceptron and 2nd-order Perceptron. For example, on Amazon3 both Perceptron and 2nd-order Perceptron have about $5.50 \%$ error when trained with no noise (Table 3 ), while having about $12 \%$ error with $10 \%$ label noise. Confidit, on the other hand, has $6.57 \%$ error with no noise, and about $8.90 \%$ with noise. Third, in fact, while Confidit performs best (among the four non margin-based algorithms) on only 3 datasets out of 9 when no noise is induced, it is the best in 4 datasets at a $10 \%$ label noise level, and is best on all 9 datasets for $20 \%$ and $30 \%$ noise. Hence Confidit seems more resilient to label noise compared to Banditron, as well as to the other two full information algorithms: Perceptron and 2nd-order Perceptron.

\subsection{Batch evaluation for vowel recognition}

Following the experimental setting in Lin et al. (2009), we now give batch evaluation results on the four vowel recognition datasets. Generally speaking, similar trends as those reported on the text categorization datasets can be observed. 

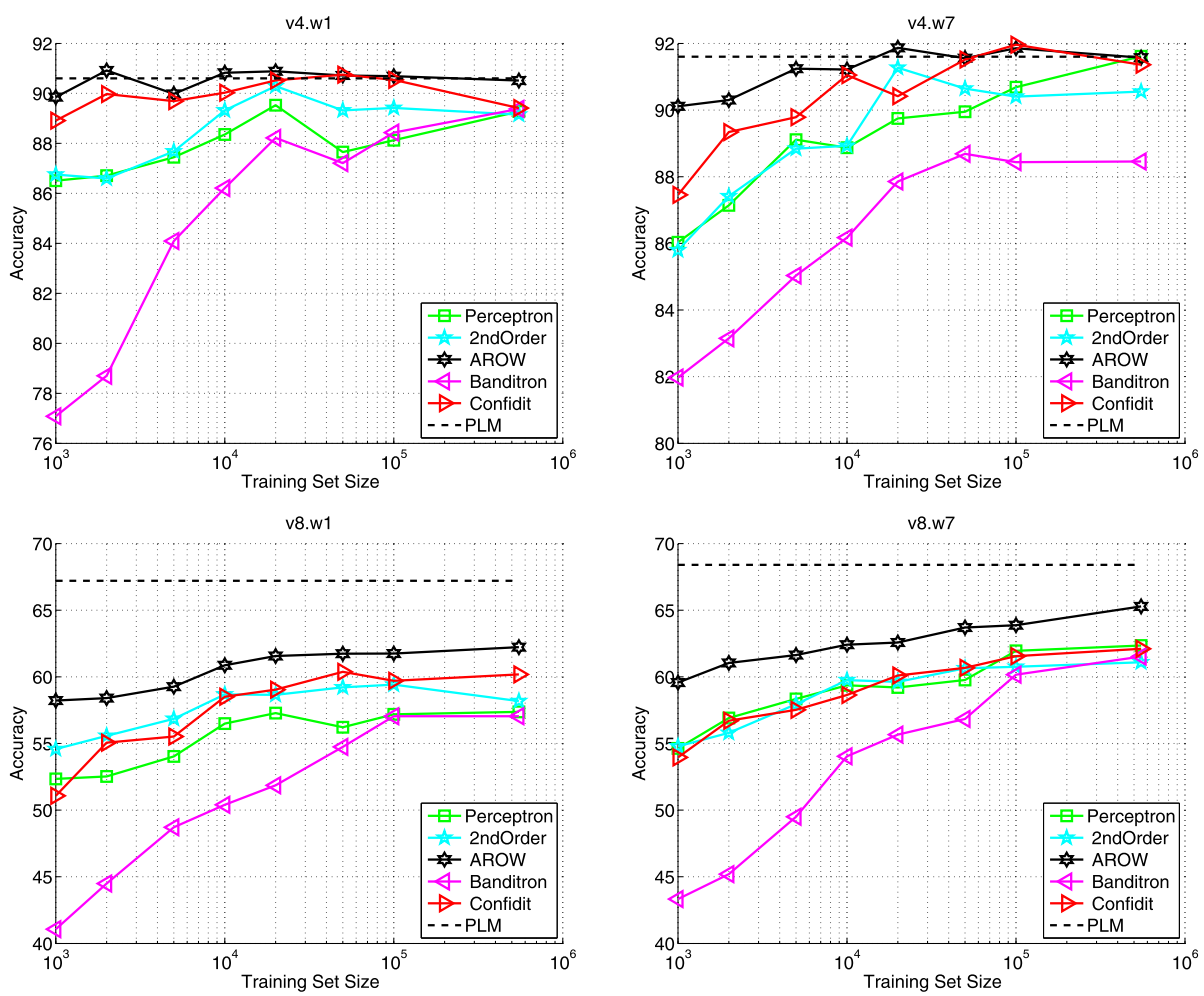

Fig. 8 Test set accuracy with no label noise on Vocal Joystick data with varying training set sizes. The online algorithms are trained on one epoch only

Test accuracies as a function of the training set size are contained in Fig. 8. All online algorithms made a single pass over the training data. The figure also includes the test set accuracy of the Probabilistic Linear Machine (PLM) algorithm (Lin et al. 2009), which achieves state-of-the-art results for that task. The PLM algorithm was trained using the entire training set. The top row shows the results for the smaller set of four labels ("v4"), and the bottom one for the larger set of eight labels ("v8"). The left column summarizes the results for one feature window ("w1"), the right column is for the seven feature window ("w7").

Focusing on the top-left panel we observe that PLM achieves $90.0 \%$ accuracy. AROW and Confidit achieve an accuracy of $89 \%$ or higher using only 1,000 examples. AROW achieves PLM's accuracy with about 2,000 training examples, and Confidit with about 20,000. The full training set size has about $275 \mathrm{~K}$ frames. All other online algorithms do not achieve this skyline, second-order Perceptron and Perceptron achieve accuracy of about $87 \%$ when trained with 1,000 examples, and reach a peak performance of about $90 \%$ (second-order perceptron) and $89 \%$ (perceptron) with about 20,000 training examples. Interestingly, all algorithms, except AROW, slightly overfit with more training data. This could be because tuning is performed with a fixed fraction of the training set, thus more training set examples are used to tune parameters when the training set is larger.

Moving to the top-right figure, when more features are available we observe a similar trend. The skyline of PLM is higher by one percent and is $91.6 \%$. Banditron's accuracy when training with 1,000 examples is $82 \%$ (higher than the accuracy of $77 \%$ with one 
frame). Then its accuracy increases to about $89 \%$ with 50,000 and the performance is not improving when more training data are available. Perceptron and second-order Perceptron start with higher accuracy of $86 \%$ when there are only 1,000 examples. Perceptron improves as more data are available until it reaches the skyline with all data. The 2nd-order Perception's accuracy is very close to the skyline with 20,000 and then starts to overfit. Confidit starts a bit higher with $87 \%$ accuracy when training with 1,000 examples, and achieves higher accuracy than all algorithms, except AROW, throughout all sizes of training set (except second-order Perceptron trained with 20,000 examples). Finally, AROW achieves the best accuracy among all online algorithms, across all datasets.

The bottom two panels show the results with the harder problems with eight labels. The skyline is lower with accuracy of $67.2 \%$ (one frame) and $68.4 \%$ (seven frames). None of the online algorithms do achieve it or get close to it. As expected, all online algorithms improve when more training data are available. In general AROW is best, then comes 2ndorder Perceptron, then Confidit, then Perceptron, and finally Banditron. The latter seems to gain most from additional data.

\section{Proofs}

This section contains the proofs of Theorem 1, Corollary 1, and Theorem 2. Specifically, Sect. 7.1 deals with Confidit and Sect. 7.4 deals with Modified Confidit.

In all cases, the analysis starts off with a one-step bound that relates the instantaneous regret of the algorithm to the current confidence level associated with the chosen class (Lemmas 1,7 , and 8). Then, it is shown that the update rule of the algorithm makes it likely for this confidence level to decrease with time. The way this is done is by controlling the quantity $\left(W_{t-1}^{\prime}{ }^{\top} X-U^{\top} X\right)^{2}$ as it evolves over time, where $W_{t-1}^{\prime}$ is the vector computed by Confidit in Step 2 (see Fig. 1), $U$ is the multiclass comparison vector, and $X$ is an arbitrary update vector. Because $X$ is arbitrary, the convergence of $W_{t-1}^{\prime}{ }^{\top} X$ to $U^{\top} X$ implies the convergence of $W_{t-1}^{\prime}$ to $U$ which, in turn, implies that the above instantaneous regret goes to zero as the number of time steps increases. The detailed rate of convergence happens to be ruled by the specific assumptions we make along the way. In particular, the convergence rate is of the form $\frac{\log T}{\sqrt{T}}$ under the assumptions of Theorem 1, it is of the form $\frac{\log ^{2} T}{T}$ under the assumptions of Corollary 1, and is dependent on the amount of noise in the more general case of Theorem 2 .

\subsection{Analysis of Confidit (Algorithm 1)}

The following standard lemma relates the error of our algorithm in computing the score values and the regret, and is a direct consequence of our learning model.

Lemma 1 If at time t the upper confidence algorithm in Eq. (4) is such that

$$
\left|\hat{\Delta}_{i, t}-\Delta_{i, t}\right| \leq \epsilon_{i, t}, \quad i=1, \ldots, K
$$

then

$$
\mathbb{P}_{t}\left(y_{t} \neq \hat{y}_{t}\right)-\mathbb{P}_{t}\left(y_{t} \neq b\left(\boldsymbol{x}_{t}\right)\right) \leq \frac{2 \epsilon_{\hat{y}_{t}, t}}{1+\alpha}
$$


Proof From (2) we can write

$$
\begin{aligned}
\mathbb{P}_{t}\left(y_{t} \neq \hat{y}_{t}\right)-\mathbb{P}_{t}\left(y_{t} \neq b\left(\boldsymbol{x}_{t}\right)\right)= & \frac{\Delta_{b_{t}, t}-\Delta_{\hat{y}_{t}, t}}{1+\alpha} \\
& \leq \frac{\hat{\Delta}_{b_{t}, t}+\epsilon_{b_{t}, t}-\hat{\Delta}_{\hat{y}_{t}, t}+\epsilon_{\hat{y}_{t}, t}}{1+\alpha} \\
& \leq \frac{\epsilon_{\hat{y}_{t}, t}-\epsilon_{b_{t}, t}+\epsilon_{b_{t}, t}+\epsilon_{\hat{y}_{t}, t}}{1+\alpha} \\
& =\frac{\left(\text { from }(4), \text { since } \hat{\Delta}_{\hat{y}_{t}, t}+\epsilon_{\hat{y}_{t}, t} \geq \hat{\Delta}_{b_{t}, t}+\epsilon_{b_{t}, t}\right)}{1+\alpha} .
\end{aligned}
$$

Let $d_{t}(U, W)$ denote the "multiclass" Mahalanobis distance $d_{t}(U, W)=$ $\frac{1}{2}(U-W)^{\top} A_{t}(U-W)$, where $U=\left(\boldsymbol{u}_{1}, \ldots, \boldsymbol{u}_{K}\right)$ and $W=\left(\boldsymbol{w}_{1}, \ldots, \boldsymbol{w}_{K}\right)$ are $d K$ dimensional column vectors and $A_{t}$ is the $t$-th $d K \times d K$-dimensional matrix produced by the algorithm. Note, $A_{t}$ is a block-diagonal matrix whose $K$ blocks are the $d \times d$ matrices $A_{1, t}, A_{2, t}, \ldots, A_{K, t}$,

$$
A_{i, t}=\left(1+\alpha^{2}\right) I+\sum_{s \leq t: \hat{y}_{s}=i} \boldsymbol{x}_{s} \boldsymbol{x}_{s}^{\top} .
$$

Let also $X_{t}$ be the $d K$-dimensional (random) vector constructed at time $t$ (Steps 6-7 of the algorithm).

Lemma 2 With the notation introduced so far, the following inequality holds for any $t$ and any $X \in \mathbb{R}^{d K}$, where we have $r_{s}=X_{s}^{\top} A_{s}^{-1} X_{s}$, and $U \in \mathbb{R}^{d K}$ is the unknown multiclass vector of parameters defined in (2).

$$
\begin{aligned}
\left(W_{t-1}^{\prime}{ }^{\top} X-U^{\top} X\right)^{2} \leq & 2 X^{\top} A_{t-1}^{-1} X\left(d_{0}(U, 0)+\frac{(1+\alpha)^{2}}{2} \sum_{s=1}^{t-1} r_{s}\right. \\
& \left.-\frac{1}{2} \sum_{s=1}^{t-1}\left(1-W_{s-1}^{\prime}{ }^{\top} X_{s}\right)^{2}+\frac{1}{2} \sum_{s=1}^{t-1}\left(1-U^{\top} X_{s}\right)^{2}\right),
\end{aligned}
$$

Proof Let $s$ be any round between round 1 and round $t-1$. From the update rule $W_{s-1}^{\prime} \rightarrow$ $W_{s}$ (Step 8 in the algorithm) we can write (see, e.g., Azoury and Warmuth 2001; Dekel et al. 2010)

$$
\begin{aligned}
& \frac{1}{2}\left(1-W_{s-1}^{\prime}{ }^{\top} X_{s}\right)^{2}-\frac{1}{2}\left(1-U^{\top} X_{s}\right)^{2} \\
& \quad=d_{s-1}\left(U, W_{s-1}^{\prime}\right)-d_{s}\left(U, W_{s}\right)+d_{s}\left(W_{s-1}^{\prime}, W_{s}\right) .
\end{aligned}
$$

Also, because $U \in C_{s}$ for any $s$, we have the standard Bregman projection inequality $d_{s}\left(U, W_{s}\right) \geq d_{s}\left(U, W_{s}^{\prime}\right)$ that we apply to (13) for $s=1, \ldots, t-1$. Finally, again using known identities (e.g., Azoury and Warmuth 2001; Dekel et al. 2010), we have

$$
d_{s}\left(W_{s-1}^{\prime}, W_{s}\right)=\frac{\left(1-W_{s-1}^{\prime} X_{s}\right)^{2}}{2} r_{s}
$$


From the algorithm pseudocode, we see that because $W_{s-1}^{\prime}$ is a projected vector, we have $\boldsymbol{w}_{\hat{y}_{s}, s-1}^{\prime}{ }^{\top} \boldsymbol{x}_{s} \in[-\alpha, 1]$. Hence $\left(1-W_{s-1}^{\prime}{ }^{\top} X_{s}\right)^{2} \leq(1+\alpha)^{2}$, implying $d_{s}\left(W_{s-1}^{\prime}, W_{s}\right) \leq(1+$ $\alpha)^{2} r_{s} / 2$. Summing over $s=1, \ldots, t-1$ and noting that $W_{0}^{\prime}=0$ yields

$$
\begin{aligned}
& \frac{1}{2} \sum_{s=1}^{t-1}\left(1-W_{s-1}^{\prime}{ }^{\top} X_{s}\right)^{2}-\frac{1}{2} \sum_{s=1}^{t-1}\left(1-U^{\top} X_{s}\right)^{2} \\
& \quad \leq d_{0}(U, 0)-d_{t-1}\left(U, W_{t-1}^{\prime}\right)+\frac{(1+\alpha)^{2}}{2} \sum_{s=1}^{t-1} r_{s} .
\end{aligned}
$$

Now, from the Cauchy-Schwarz inequality (for dual norms) we have

$$
\left(W_{t-1}^{\prime}{ }^{\top} X-U^{\top} X\right)^{2} \leq 2 X^{\top} A_{t-1}^{-1} X d_{t-1}\left(U, W_{t-1}^{\prime}\right) .
$$

Solving (14) for $d_{t-1}\left(U, W_{t-1}^{\prime}\right)$ and plugging back into the last inequality concludes the proof.

The next three lemmas are intended to control with high probability the cumulative difference of square losses

$$
\sum_{s=1}^{t-1} D_{s}, \quad \text { with } D_{s}=\frac{1}{2}\left(1-W_{s-1}^{\prime}{ }^{\top} X_{s}\right)^{2}-\frac{1}{2}\left(1-U^{\top} X_{s}\right)^{2},
$$

occurring in Lemma 2. We do this in three steps. First, we prove that, conditioned on the past, the expectation of $D_{s}$ cannot be negative (Lemma 3). Then we show that the conditional variance of $D_{s}$ is close to its conditional expectation (Lemma 4). Finally, we apply a standard martingale concentration argument to prove that with high probability $\sum_{s=1}^{t-1} D_{s}$ cannot be too negative. Specifically, $\sum_{s=1}^{t-1} D_{s}$ cannot decrease more than logarithmically with $t$ (Lemma 5).

Recall that $E_{t}[\cdot]$ denotes the conditional expectation $E\left[\cdot \mid \boldsymbol{x}_{1}, \ldots \boldsymbol{x}_{t}, y_{1}, \ldots y_{t-1}, \sigma_{t-1}\right]$, the expectation being over the current random label $y_{t}$ as given in (2) and the random choices of the algorithm in round $t$. Similarly, let $\operatorname{Var}_{t}(\cdot)$ denote the conditional variance.

Lemma 3 With the notation introduced so far, the following inequality holds for any (constant) $W=\left(\boldsymbol{w}_{1}, \ldots, \boldsymbol{w}_{K}\right) \in \mathbb{R}^{d K}$, where $U \in \mathbb{R}^{d K}$ is the multiclass vector of parameters defined in (2):

$$
E_{t}\left[\frac{1}{2}\left(1-W^{\top} X_{t}\right)^{2}-\frac{1}{2}\left(1-U^{\top} X_{t}\right)^{2}\right]=\frac{1}{2}\left(\boldsymbol{u}_{\hat{y}_{t}}^{\top} \boldsymbol{x}_{t}-\boldsymbol{w}_{\hat{y}_{t}}^{\top} \boldsymbol{x}_{t}\right)^{2} \geq 0
$$

Proof Let us introduce the shorthands $\Delta=\boldsymbol{u}_{\hat{y}_{t}}^{\top} \boldsymbol{x}_{t}, \hat{\Delta}=\boldsymbol{w}_{\hat{y}_{t}}^{\top} \boldsymbol{x}_{t}, p=\frac{\alpha+\Delta}{1+\alpha}$, and $\gamma=\frac{1+\alpha}{2}$. From Steps 6 and 7 of the algorithm and Eq. (2) we can write

$$
E_{t}\left[\left(1-W^{\top} X_{t}\right)^{2}\right]=((1-p)(1-\gamma)+p)(1-\hat{\Delta})^{2}+(1-p) \gamma(1+\hat{\Delta})^{2} .
$$

Similarly,

$$
E_{t}\left[\left(1-U^{\top} X_{t}\right)^{2}\right]=((1-p)(1-\gamma)+p)(1-\Delta)^{2}+(1-p) \gamma(1+\Delta)^{2} .
$$


Hence, recalling $p$ and $\gamma$, we have

$$
\begin{aligned}
E_{t} & {\left[\left(1-W^{\top} X_{t}\right)^{2}-\left(1-U^{\top} X_{t}\right)^{2}\right] } \\
& =((1-p)(1-\gamma)+p)\left((1-\hat{\Delta})^{2}-(1-\Delta)^{2}\right)+(1-p) \gamma\left((1+\hat{\Delta})^{2}-(1+\Delta)^{2}\right) \\
& =((1-p)(1-\gamma)+p)(\hat{\Delta}+\Delta-2)(\hat{\Delta}-\Delta)+(1-p) \gamma(2+\hat{\Delta}+\Delta)(\hat{\Delta}-\Delta) \\
& =\left(\frac{1+\Delta}{2}\right)(\hat{\Delta}+\Delta-2)(\hat{\Delta}-\Delta)+\left(\frac{1-\Delta}{2}\right)(2+\hat{\Delta}+\Delta)(\hat{\Delta}-\Delta) \\
& =(\Delta-\hat{\Delta})^{2} .
\end{aligned}
$$

Lemma 4 With the notation introduced so far we have, for any (constant) $W \in C_{t}$,

$$
\operatorname{Var}_{t}\left[\frac{1}{2}\left(1-W^{\top} X_{t}\right)^{2}-\frac{1}{2}\left(1-U^{\top} X_{t}\right)^{2}\right] \leq(1+\alpha)^{2}\left(\boldsymbol{u}_{\hat{y}_{t}}^{\top} \boldsymbol{x}_{t}-\boldsymbol{w}_{\hat{y}_{t}}^{\top} \boldsymbol{x}_{t}\right)^{2},
$$

where $U \in \mathbb{R}^{d K}$ is the multiclass vector of parameters defined in (2).

Proof We slightly overload the notation and set within this proof $D_{t}=\frac{1}{2}\left(1-W^{\top} X_{t}\right)^{2}-$ $\frac{1}{2}\left(1-U^{\top} X_{t}\right)^{2}$. We can write

$$
\begin{aligned}
D_{t} & =U^{\top} X_{t}-W^{\top} X_{t}+\frac{1}{2}\left(W^{\top} X_{t}\right)^{2}-\frac{1}{2}\left(U^{\top} X_{t}\right)^{2} \\
& =\left(U^{\top} X_{t}-W^{\top} X_{t}\right)\left(2-W^{\top} X_{t}-U^{\top} X_{t}\right) / 2 .
\end{aligned}
$$

Now, $\operatorname{Var}_{t}\left[D_{t}\right]=E_{t}\left[D_{t}^{2}\right]-E_{t}^{2}\left[D_{t}\right] \leq E_{t}\left[D_{t}^{2}\right]$, where

$$
\begin{aligned}
E_{t}\left[D_{t}^{2}\right] & =E_{t}\left[\left(U^{\top} X_{t}-W^{\top} X_{t}\right)^{2}\left(2-W^{\top} X_{t}-U^{\top} X_{t}\right)^{2} / 4\right] \\
& \leq(1+\alpha)^{2} E_{t}\left[\left(U^{\top} X_{t}-W^{\top} X_{t}\right)^{2}\right] \\
& \left(\text { since } W \in C_{t} \text { implies }\left|\frac{2-W^{\top} X_{t}-U^{\top} X_{t}}{2}\right| \leq 1+\alpha\right) \\
& =(1+\alpha)^{2}\left(\boldsymbol{u}_{\hat{y}_{t}}^{\top} \boldsymbol{x}_{t}-\boldsymbol{w}_{\hat{y}_{t}}^{\top} \boldsymbol{x}_{t}\right)^{2} .
\end{aligned}
$$

Note that in order to bound the variance, we require the vector $W$ to belong to the set $C_{t}$. This is the motivation and need for the projection step of the algorithm (step 2 of Fig. 1).

Lemma 5 With the notation introduced so far we have

$$
\mathbb{P}\left(\sum_{s=1}^{t-1} D_{s} \geq-9(1+\alpha)^{2} \log \frac{t+4}{\delta}\right) \geq 1-\frac{\delta}{t(t+1)},
$$

where $W_{0}^{\prime}, W_{1}^{\prime}, \ldots, W_{t-2}^{\prime}$ in $D_{1}, \ldots, D_{t-1}$ is the sequence of projected weight vectors produced by the algorithm at the beginning of rounds $1,2, \ldots, t-1$, respectively.

Proof Let $D_{s}^{\prime}=E_{s}\left[D_{s}\right]-D_{s}$. Then the sequence $D_{1}^{\prime}, D_{2}^{\prime}, \ldots, D_{t-1}^{\prime}$ is a martingale difference sequence such that: 
(i) $\left|D_{s}^{\prime}\right| \leq(1+\alpha)^{2}$ (since $\left.U, W_{s-1}^{\prime} \in C_{s}\right)$,

(ii) $E_{s}\left[D_{s}\right]=\frac{1}{2}\left(\boldsymbol{u}_{\hat{y}_{s}}^{\top} \boldsymbol{x}_{s}-\boldsymbol{w}_{\hat{y}_{s}, s-1}^{\prime \top} \boldsymbol{x}_{s}\right)^{2}$ (because of Lemma 3), and

(iii) $\operatorname{Var}_{s}\left[D_{s}^{\prime}\right]=\operatorname{Var}_{s}\left[D_{s}\right] \leq(1+\alpha)^{2}\left(\boldsymbol{u}_{\hat{y}_{s}}^{\top} \boldsymbol{x}_{s}-\boldsymbol{w}_{\hat{y}_{s}, s-1}^{\prime \top} \boldsymbol{x}_{s}\right)^{2}$ (because of Lemma 4).

We are then in a position to apply any concentration result on bounded martingale difference sequences. Set for brevity

$$
\mu_{s}=\left(\boldsymbol{u}_{\hat{y}_{s}}^{\top} \boldsymbol{x}_{s}-\boldsymbol{w}_{\hat{y}_{s}, s-1}^{\top} \boldsymbol{x}_{s}\right)^{2}, \quad s=1, \ldots, t-1 .
$$

From Kakade and Tewari (2008), we can see that when $t \geq 4$

$$
\begin{aligned}
& \sum_{s=1}^{t-1} \frac{1}{2} \mu_{s}-\sum_{s=1}^{t-1} D_{s} \\
& \quad \geq \max \left\{\sqrt{\left.4(1+\alpha)^{2} \log \frac{4 t(t+1) \log (t-1)}{\delta} \sum_{s=1}^{t-1} \mu_{s}, 3(1+\alpha)^{2} \log \frac{4 t(t+1) \log (t-1)}{\delta}\right\}}\right.
\end{aligned}
$$

holds with probability at most $\frac{\delta}{t(t+1)}$. We use the inequality $\sqrt{c b} \leq \frac{1}{2}(c+b)$ with $c=4(1+$ $\alpha)^{2} \log \frac{4 t(t+1) \log (t-1)}{\delta}$, and $b=\sum_{s=1}^{t-1} \mu_{s}$ to conclude that with probability at least $1-\frac{\delta}{t(t+1)}$ we have

$$
\sum_{s=1}^{t-1} D_{s} \geq-3(1+\alpha)^{2} \log \frac{4 t(t+1) \log (t-1)}{\delta} .
$$

A further overapproximation to incorporate the condition $t \geq 4$ concludes the proof.

Combining Lemmas 2 through 5 we get the following approximation result, which delivers the announced upper confidence levels $\epsilon_{i, t}$ in (9).

Lemma 6 With the notation introduced so far we have that

$$
\begin{aligned}
\left(\hat{\Delta}_{i, t}^{\prime}-\Delta_{i, t}\right)^{2} \leq & 2 \boldsymbol{x}_{t}^{\top} A_{i, t-1}^{-1} \boldsymbol{x}_{t}\left(\frac{1}{2}(1+\alpha)^{2}\|U\|_{2}^{2}\right. \\
& \left.+\frac{(1+\alpha)^{2}}{2} \sum_{s=1}^{t-1} X_{s}^{\top} A_{s}^{-1} X_{s}+9(1+\alpha)^{2} \log \frac{t+4}{\delta}\right)
\end{aligned}
$$

holds with probability at least $1-\delta$, uniformly over $i=1, \ldots, K$ and $t=1,2, \ldots$.

Proof Combining Lemma 2 with Lemma 5 gives

$$
\begin{aligned}
\left(W_{t-1}^{\prime}{ }^{\top} X-U^{\top} X\right)^{2} \leq & 2 X^{\top} A_{t-1}^{-1} X\left(d_{0}(U, 0)\right. \\
& \left.+\frac{(1+\alpha)^{2}}{2} \sum_{s=1}^{t-1} X_{s}^{\top} A_{s}^{-1} X_{s}+9(1+\alpha)^{2} \log \frac{t+4}{\delta}\right)
\end{aligned}
$$


holding with probability at least $1-\frac{\delta}{t(t+1)}$ uniformly over $X$. Recall that $d_{0}(U, 0)=$ $\frac{1}{2}(1+\alpha)^{2}\|U\|_{2}^{2}$. In the above we set $X=\left(\boldsymbol{x}_{t}, 0, \ldots, 0\right), X=\left(0, \boldsymbol{x}_{t}, 0, \ldots, 0\right), \ldots, X=$ $\left(0, \ldots, 0, \boldsymbol{x}_{t}\right)$, and take a union bound over $t$. Recalling (12) proves the lemma.

\subsection{Proof of Theorem 1}

From Lemma 1 and Lemma 6 we have, with probability at least $1-\delta$,

$$
\sum_{t=1}^{T}\left(\mathbb{P}_{t}\left(y_{t} \neq \hat{y}_{t}\right)-\mathbb{P}_{t}\left(y_{t} \neq b\left(\boldsymbol{x}_{t}\right)\right)\right) \leq \frac{2}{1+\alpha} \sum_{t=1}^{T} \epsilon_{\hat{y}_{t}, t},
$$

where $\epsilon_{\hat{y}_{t}, t}$ satisfies (9). Hence we continue to derive a pointwise upper bound on the sum in the RHS.

Following previous techniques (e.g. Azoury and Warmuth 2001; Cesa-Bianchi et al. 2005), we have

$$
\begin{aligned}
\sum_{s=1}^{t-1} X_{s}^{\top} A_{s}^{-1} X_{s} & \leq \log \frac{\left|A_{t-1}\right|}{\left|A_{0}\right|} \\
& =\sum_{i=1}^{K} \log \frac{\left|A_{i, t-1}\right|}{\left|A_{i, 0}\right|} \\
& =B_{3} \\
& \leq \sum_{i=1}^{K} d \log \left(1+\frac{N_{i, t-1}}{d(1+\alpha)^{2}}\right) \\
& \leq d K \log \left(1+\frac{T}{d K(1+\alpha)^{2}}\right)
\end{aligned}
$$

where $N_{i, t-1}$ is the number of times class $i$ has been chosen by the algorithm up to time $t-1$. Now observe that when the algorithm predicts with class $\hat{y}_{t}$ at time $t$ then the corresponding matrix $A_{\hat{y}_{t}, t-1}$ used at the beginning of round $t$ gets augmented by the rank-one matrix $\boldsymbol{x}_{t} \boldsymbol{x}_{t}^{\top}$, independent of the value of $M_{t}$. Hence, $\boldsymbol{x}_{t}^{\top} A_{\hat{y}_{t}, t-1}^{-1} \boldsymbol{x}_{t} \leq(1+\alpha)^{-2}$ allows us to write $1 \leq\left(1+(1+\alpha)^{-2}\right) /\left(1+\boldsymbol{x}_{t}^{\top} A_{\hat{y}_{t}, t-1}^{-1} \boldsymbol{x}_{t}\right)$ and thus,

$$
\boldsymbol{x}_{t}^{\top} A_{\hat{y}_{t}, t-1}^{-1} \boldsymbol{x}_{t} \leq \frac{\left(1+(1+\alpha)^{-2}\right) \boldsymbol{x}_{t}^{\top} A_{\hat{y}_{t}, t-1}^{-1} \boldsymbol{x}_{t}}{1+\boldsymbol{x}_{t}^{\top} A_{\hat{y}_{t}, t-1}^{-1} \boldsymbol{x}_{t}}=\left(1+(1+\alpha)^{-2}\right) \boldsymbol{x}_{t}^{\top} A_{\hat{y}_{t}, t}^{-1} \boldsymbol{x}_{t},
$$

the equality following from the Sherman-Morrison matrix inversion formula. Setting for brevity $C=(1+\alpha)^{2}\left(B_{2}+B_{3}\right)$, we write

$$
\sum_{t=1}^{T} \epsilon_{\hat{y}_{t}, t}^{2} \leq C \sum_{t=1}^{T} \boldsymbol{x}_{t}^{\top} A_{\hat{y}_{t}, t-1}^{-1} \boldsymbol{x}_{t} \leq C\left(1+(1+\alpha)^{-2}\right) \sum_{t=1}^{T} \boldsymbol{x}_{t}^{\top} A_{\hat{y}_{t}, t}^{-1} \boldsymbol{x}_{t} .
$$

Using again the same argument as above we can upper bound the last sum by $B_{3}$. Hence

$$
\sum_{t=1}^{T} \epsilon_{\hat{y}_{t}, t}^{2} \leq\left(1+(1+\alpha)^{2}\right)\left(B_{2}+B_{3}\right) B_{3} .
$$


Finally, since $\sum_{t=1}^{T} \epsilon_{\hat{y}_{t}, t}^{2} \leq M$ implies $\sum_{t=1}^{T} \epsilon_{\hat{y}_{t}, t} \leq \sqrt{T M}$, putting together as in (16) and overapproximating concludes the proof.

\subsection{Proof of Corollary 1}

The low-noise argument replaces Lemma 1 by the following.

Lemma 7 Let $\epsilon>0$ be any constant. If at time $t$ the upper confidence algorithm in Eq. (4) is such that

$$
\left|\hat{\Delta}_{i, t}-\Delta_{i, t}\right| \leq \epsilon_{i, t} \quad i=1, \ldots, K
$$

then

$$
\mathbb{P}_{t}\left(y_{t} \neq \hat{y}_{t}\right)-\mathbb{P}_{t}\left(y_{t} \neq b\left(\boldsymbol{x}_{t}\right)\right) \leq\left\{\Delta_{b_{t}, t}-\Delta_{\hat{y}_{t}, t} \leq \epsilon\right\} \frac{\epsilon}{1+\alpha}+\frac{4 \epsilon_{\hat{y}_{t}, t}^{2}}{(1+\alpha) \epsilon}
$$

Proof From (2) we write

$$
\begin{aligned}
\mathbb{P}_{t}\left(y_{t} \neq \hat{y}_{t}\right)-\mathbb{P}_{t}\left(y_{t} \neq b\left(\boldsymbol{x}_{t}\right)\right) \\
=\frac{\Delta_{b_{t}, t}-\Delta_{\hat{y}_{t}, t}}{1+\alpha} \\
=\left\{\Delta_{b_{t}, t}-\Delta_{\hat{y}_{t}, t} \leq \epsilon\right\} \frac{\Delta_{b_{t}, t}-\Delta_{\hat{y}_{t}, t}}{1+\alpha}+\left\{\Delta_{b_{t}, t}-\Delta_{\hat{y}_{t}, t}>\epsilon\right\} \frac{\Delta_{b_{t}, t}-\Delta_{\hat{y}_{t}, t}}{1+\alpha} \\
\quad \leq\left\{\Delta_{b_{t}, t}-\Delta_{\hat{y}_{t}, t} \leq \epsilon\right\} \frac{\epsilon}{1+\alpha}+\frac{\left(\Delta_{b_{t}, t}-\Delta_{\hat{y}_{t}, t}\right)^{2}}{(1+\alpha) \epsilon} \\
\quad \leq\left\{\Delta_{b_{t}, t}-\Delta_{\hat{y}_{t}, t} \leq \epsilon\right\} \frac{\epsilon}{1+\alpha}+\frac{\left(\hat{\Delta}_{b_{t}, t}+\epsilon_{b_{t}, t}-\hat{\Delta}_{\hat{y}_{t}, t}+\epsilon_{\hat{y}_{t}, t}\right)^{2}}{(1+\alpha) \epsilon} \\
\leq\left\{\Delta_{b_{t}, t}-\Delta_{\hat{y}_{t}, t} \leq \epsilon\right\} \frac{\epsilon}{1+\alpha}+\frac{\left(\epsilon_{\hat{y}_{t}, t}-\epsilon_{b_{t}, t}+\epsilon_{b_{t}, t}+\epsilon_{\hat{y}_{t}, t}\right)^{2}}{(1+\alpha) \epsilon} \\
=\left\{\Delta_{b_{t}, t}-\Delta_{\hat{y}_{t}, t} \leq \epsilon\right\} \frac{\epsilon}{1+\alpha}+\frac{4 \epsilon_{\hat{y}_{t}, t}^{2}}{(1+\alpha) \epsilon}
\end{aligned}
$$

thereby concluding the proof.

To conclude the proof of Corollary 1, it suffices to combine Lemmas 1 through 6 as in the proof of Theorem 1 by using Eq. (18).

\subsection{Analysis of Modified Confidit}

We need to generalize Lemmas 1 through 6. In the sequel, we only stress how to adapt their proofs. First, Lemma 1 is generalized as follows.

Lemma 8 Let $q_{t}$ be the time-t approximation error. If at time t the upper confidence algorithm in Eq. (4) is such that

$$
\left|\hat{\Delta}_{i, t}-\Delta_{i, t}\right| \leq \epsilon_{i, t}, \quad i=1, \ldots, K,
$$


then

$$
\mathbb{P}_{t}\left(y_{t} \neq \hat{y}_{t}\right)-\mathbb{P}_{t}\left(y_{t} \neq b\left(\boldsymbol{x}_{t}\right)\right) \leq 2 q_{t}+\frac{2 \epsilon_{\hat{y}_{t}, t}}{1+\alpha}
$$

Proof From the definition of $q_{t}$ we can write

$$
\begin{aligned}
\mathbb{P}_{t}\left(y_{t} \neq \hat{y}_{t}\right)-\mathbb{P}_{t}\left(y_{t} \neq b\left(\boldsymbol{x}_{t}\right)\right) & =\mathbb{P}_{t}\left(y_{t}=b_{t}\right)-\mathbb{P}_{t}\left(y_{t}=\hat{y}_{t}\right) \\
& \leq q_{t}+\frac{\alpha+\Delta_{b_{t}, t}}{1+\alpha}+q_{t}-\frac{\alpha+\Delta_{\hat{y}_{t}, t}}{1+\alpha} \\
& =2 q_{t}+\frac{\Delta_{b_{t}, t}-\Delta_{\hat{y}_{t}, t}}{1+\alpha} .
\end{aligned}
$$

Then we continue to bound the last term just as in the proof of Lemma 1.

Lemma 2 only depends on the update rule of the algorithm, hence it holds unchanged for Modified Confidit as well. Next, we adapt Lemma 3.

Lemma 9 With the notation introduced so far,

$$
\begin{aligned}
& E_{t}\left[\frac{1}{2}\left(1-W^{\top} X_{t}\right)^{2}-\frac{1}{2}\left(1-U^{\top} X_{t}\right)^{2}\right] \\
& \quad \geq \frac{1}{2}\left(\boldsymbol{u}_{\hat{y}_{t}}^{\top} \boldsymbol{x}_{t}-\boldsymbol{w}_{\hat{y}_{t}}^{\top} \boldsymbol{x}_{t}\right)^{2}-(1+\alpha)\left|\boldsymbol{u}_{\hat{y}_{t}}^{\top} \boldsymbol{x}_{t}-\boldsymbol{w}_{\hat{y}_{t}}^{\top} \boldsymbol{x}_{t}\right| q_{t}
\end{aligned}
$$

holds for any (constant) $W=\left(\boldsymbol{w}_{1}, \ldots, \boldsymbol{w}_{K}\right) \in \mathbb{R}^{d K}$, where $U \in \mathbb{R}^{d K}$ is the multiclass vector of parameters defined in (2).

Proof We use the same shorthands as in the proof of Lemma 3, where now $p=\mathbb{P}\left(y_{t}=\right.$ $\left.\hat{y}_{t} \mid \boldsymbol{x}_{t}\right)$. Following the same steps therein we can see that

$$
E_{t}\left[\left(1-W^{\top} X_{t}\right)^{2}-\left(1-U^{\top} X_{t}\right)^{2}\right]=(\hat{\Delta}-\Delta)(\hat{\Delta}+\Delta-2+4 \gamma)-(\hat{\Delta}-\Delta) 4 p \gamma .
$$

Now, the approximation bound $\left|p-\frac{\alpha+\Delta}{1+\alpha}\right| \leq q_{t}$ implies that the RHS is at least $(\hat{\Delta}-\Delta)^{2}-$ $4 \gamma|\hat{\Delta}-\Delta| q_{t}$, thereby concluding the proof.

The proof of Lemma 4 is not affected by the modified class conditional distribution, hence it holds unchanged. The next lemma combines Lemma 9 and Lemma 4 into the following more general concentration bound. Recall that $D_{s}=\frac{1}{2}\left(1-W_{s-1}^{\prime}{ }^{\top} X_{s}\right)^{2}$.

Lemma 10 With the notation introduced so far we have

$$
\mathbb{P}\left(\sum_{s=1}^{t-1} D_{s} \geq-(1+\alpha)^{2} \sum_{s=1}^{t-1} q_{s}-9(1+\alpha)^{2} \log \frac{t+4}{\delta}\right) \geq 1-\frac{\delta}{t(t+1)},
$$

where $W_{0}^{\prime}, W_{1}^{\prime}, \ldots, W_{t-2}^{\prime}$ in $D_{1}, \ldots, D_{t-1}$ is the sequence of projected weight vectors produced by Modified Confidit at the beginning of rounds $1,2, \ldots, t-1$, respectively. 
Proof We follow the very same steps as in the proof of Lemma 5, except that now, from Lemma 9,

$$
\begin{aligned}
E_{s}\left[D_{s}\right] & \geq \frac{1}{2}\left(\boldsymbol{u}_{\hat{y}_{s}}^{\top} \boldsymbol{x}_{s}-\boldsymbol{w}_{\hat{y}_{s}, s-1}^{\prime \top} \boldsymbol{x}_{s}\right)^{2}-(1+\alpha)\left|\boldsymbol{u}_{\hat{y}_{s}}^{\top} \boldsymbol{x}_{s}-\boldsymbol{x}_{s}^{\top} \boldsymbol{w}_{\hat{y}_{s}}^{\prime}\right| q_{s} \\
& \geq \frac{1}{2}\left(\boldsymbol{u}_{\hat{y}_{s}}^{\top} \boldsymbol{x}_{s}-\boldsymbol{w}_{\hat{y}_{s}, s-1}^{\prime} \boldsymbol{x}_{s}\right)^{2}-(1+\alpha)^{2} q_{s},
\end{aligned}
$$

the last inequality following from the fact that $\boldsymbol{u}_{\hat{y}_{s}}^{\top} \boldsymbol{x}_{s}$ and $\boldsymbol{w}_{\hat{y}_{s}, s-1}^{\prime \top} \boldsymbol{x}_{s}$ are both in $[-\alpha, 1]$.

Lemma 6 turns into the following approximation bound, resulting from the combination of Lemma 2, Lemma 9, Lemma 4, and Lemma 10. It is this approximation that provides the upper confidence level $\epsilon_{i, t}$ contained in (10).

Lemma 11 With the notation introduced so far we have that

$$
\begin{aligned}
\left(\hat{\Delta}_{i, t}^{\prime}-\Delta_{i, t}\right)^{2} \leq & 2 \boldsymbol{x}_{t}^{\top} A_{i, t-1}^{-1} \boldsymbol{x}_{t}\left(\frac{1}{2}(1+\alpha)^{2}\|U\|_{2}^{2}\right. \\
& \left.+\frac{(1+\alpha)^{2}}{2} \sum_{s=1}^{t-1} X_{s}^{\top} A_{s}^{-1} X_{s}+(1+\alpha)^{2} \sum_{s=1}^{t-1} q_{s}+9(1+\alpha)^{2} \log \frac{t+4}{\delta}\right)
\end{aligned}
$$

holds with probability at least $1-\delta$, uniformly over $i=1, \ldots, K$ and $t=1,2, \ldots$

\subsection{Proof of Theorem 2}

From Lemma 8 and Lemma 11 we have, with probability at least $1-\delta$,

$$
\sum_{t=1}^{T}\left(\mathbb{P}_{t}\left(y_{t} \neq \hat{y}_{t}\right)-\mathbb{P}_{t}\left(y_{t} \neq b\left(\boldsymbol{x}_{t}\right)\right)\right) \leq 2 \sum_{t=1}^{T} q_{t}+\frac{2}{1+\alpha} \sum_{t=1}^{T} \epsilon_{\hat{y}_{t}, t},
$$

where $\epsilon_{\hat{y}_{t}, t}$ satisfies (10). Continuing as in the proof of Theorem 1 leads to the desired statement.

\section{Conclusions and open problems}

We presented the Confidit algorithm, which is based on combining 2nd-order Perceptron for multiclass problems and upper confidence bounds. We provided a regret bound of $\mathcal{O}(\sqrt{T} \log T)$ in a partial adversarial setting, which improves in our setting on the $T^{2 / 3}$ bound proven for the Banditron algorithm. We have also sketched an extended analysis where no assumptions whatsoever are made on the conditional distribution of the labels, and a (cumulative) approximation error has to be taken into account.

Our algorithm can also be viewed as maintaining a similar model to that of AROW (Crammer et al. 2009b), where for each label we keep a Gaussian distribution over models. Given an input example the algorithm includes a one-dimensional Gaussian distribution over the per-label margin, and outputs the label with the largest combination of means and standard deviation, the tradeoff being computed in Eq. (9). 
Experiments with an efficient version of the algorithm indicate the superiority of our algorithm, especially against mistake-driven algorithms that work both in the bandit and the full information setting. Our results on 13 datasets, both for text classification and speech recognition (vowel classification) indicate that the generalization ability of our algorithms is sometimes better than of algorithms that use full information. We leave the analysis of this phenomenon to future work.

Slightly after our paper was prepared, a related paper (Valizadegan et al. 2011) became available which contains a more involved version of the Banditron algorithm. This is essentially a Banditron algorithm whose exploration rate depends on the past behavior of the algorithm (rather than being constant). Though (Valizadegan et al. 2011) report improved experimental results over the standard Banditron, no relevant theoretical enhancements are provided.

Another very recent work that was published after our paper was submitted is (Hazan and Kale 2011). The authors describe a second-order descent algorithm for the multiclass problem with partial information. Like the Banditron, this algorithm works in the fullyadversarial setting but, unlike ours, their notion of regret is based on the log-loss, rather than the number of mistakes, which we find the direct quantity of interest.

Our current analysis of Confidit is not capturing the right dependence on the $\alpha$ parameter. In fact, one would expect the bounds to improve as $\alpha$ gets closer to -1 . The technical difficulty stems from Eq. (17), where an inverse dependence on $(1+\alpha)^{2}$ is derived when passing from $\boldsymbol{x}_{t}^{\top} A_{\hat{y}_{t}, t-1}^{-1} \boldsymbol{x}_{t}$ to $\boldsymbol{x}_{t}^{\top} A_{\hat{y}_{t}, t}^{-1} \boldsymbol{x}_{t}$.

Another open question is to provide a sharper analysis of Modified Confidit that makes further assumptions on the class conditional distributions $\mathbb{P}(y=i \mid \boldsymbol{x})$. For instance, if $\mathbb{P}\left(y_{t}=i \mid \boldsymbol{x}_{t}\right)$ is a Lipschitz function of $\Delta_{i, t}$ then the one-step regret in Lemma 8 will be (at most) proportional to $\left|\Delta_{b_{t}, t}-\Delta_{\hat{y}_{t}, t}\right|$, and the deviation terms $q_{t}$ will only occur in the bias handled by Lemma 9. This might lead to improved bounds, upon proper changes in the martingale construction of Lemma 10.

Finally, a broader open question is developing a margin-based version of the algorithm, as such algorithms often outperform mistake-driven algorithms in practice.

Acknowledgements We thank the anonymous reviewer for useful comments that helped us to significantly improve the presentation of this paper. This research was done while Claudio Gentile was visiting the Technion. This work was partly supported by the PASCAL2 Network of Excellence under EC grant no. 216886 and partly by the Israeli Science Foundation grant ISF-1567/10. This publication only reflects the authors' views. Koby Crammer is a Horev fellow supported by the Taub foundations.

\section{References}

Auer, P. (2003). Using confidence bounds for exploitation-exploration trade-offs. Journal of Machine Learning Research, 3, 397-422.

Azoury, K. S., \& Warmuth, M. K. (2001). Relative loss bounds for online density estimation with the exponential family of distributions. Machine Learning, 43, 211-246.

Blitzer, J., Dredze, M., \& Pereira, F. (2007). Biographies, Bollywood, boom-boxes and blenders: domain adaptation for sentiment classification. In Association of computational linguistics (ACL).

Cesa-Bianchi, N., Conconi, A., \& Gentile, C. (2005). A second-order perceptron algorithm. SIAM Journal on Computing, 43, 640-668.

Cesa-Bianchi, N., Gentile, C., \& Orabona, F. (2009). Robust bounds for classification via selective sampling. In Proc. 26th ICML.

Crammer, K., \& Singer, Y. (2002). On the learnability and design of output codes for multiclass problems. Machine Learning, 47, 201-233.

Crammer, K., \& Singer, Y. (2003). Ultraconservative online algorithms for multiclass problems. Journal of Machine Learning Research, 3, 951-991. 
Crammer, K., Dredze, M., \& Kulesza, A. (2009a). Multi-class confidence weighted algorithms. In EMNLP 2009.

Crammer, K., Kulesza, A., \& Dredze, M. (2009b). Adaptive regularization of weighted vectors. In Nips 2009.

Dani, V., Hayes, T., \& Kakade, S. (2008). Stochastic linear optimization under bandit feedback. In Colt 2008.

Dekel, O., Gentile, C., \& Sridharan, K. (2010). Robust selective sampling from single and multiple teachers. In Colt 2010.

Dredze, M., Crammer, K., \& Pereira, F. (2008). Confidence-weighted linear classification. In ICML 2008.

Duchi, J., Shalev-Shwartz, S., Singer, Y., \& Chandra, T. (2008). Efficient projections onto the $\ell_{1}$-ball for learning in high dimensions. In ICML 2008 (pp. 272-279).

Hazan, E., \& Kale, S. (2011). Newtron: an efficient bandit algorithm for online multiclass prediction. In NIPS 2011.

Hoerl, A., \& Kennard, R. (1970). Ridge regression: biased estimation for nonorthogonal problems. Technometrics, 12, 55-67.

Kakade, S., \& Tewari, A. (2008). On the generalization ability of online strongly convex programming algorithm. In Nips 2008.

Kakade, S., Shalev-Shwartz, S., \& Tewari, A. (2008). Efficient bandit algorithms for online multiclass prediction. In ICML 2008.

Kilanski, K., Malkin, J., Li, X., Wright, R., \& Bilmes, J. (2006). The Vocal Joystick data collection effort and vowel corpus. In Interspeech, Pittsburgh, PA.

Langford, J., \& Zhang, T. (2007). The epoch-greedy algorithm for contextual multi-armed bandits. In Nips 2007.

Lewis, D. D., Yang, Y., Rose, T. G., \& Li, F. (2004). Rcv1: a new benchmark collection for text categorization research. Journal of Machine Learning Research, 5, 361-397.

Lin, H., Bilmes, J., \& Crammer, K. (2009). How to loose confidence: probabilistic linear machines for multiclass classification. In INTERSPEECH (pp. 2559-2562).

Liu, J., \& Ye, J. (2009). Efficient Euclidean projections in linear time. In ICML 2009 (p. 83).

Lu, T., Pal, D., \& Pal, M. (2010). Showing relevant ads via Lipschitz context multi-armed bandits. In Aistat 2010.

Rigollet, P., \& Zeevi, A. (2010). Nonparametric bandits with covariates. In Colt 2010.

Rosenblatt, F. (1958). The perceptron: A probabilistic model for information storage and organization in the brain. Psychological Review, 65, 386-407.

Sandhaus, E. (2008). The New York Times annotated corpus. Philadelphia: Linguistic Data Consortium.

Strehl, A., \& Littman, M. (2008). Online linear regression and its application to model-based reinforcement learning. In NIPS 2008.

Valizadegan, H., Jin, R., \& Wang, S. (2011). Learning to trade off between exploration and exploitation in multiclass bandit prediction. In KDD 2011.

Walsh, T. J., Szita, I., Diuk, C., \& Littman, M. L. (2009). Exploring compact reinforcement-learning representations with linear regression. In UAI 2008 \& Rutgers Univ. Tech. Rep.

Wang, C., Kulkarni, S., \& Poor, V. (2005). Bandit problems with side observation. IEEE Transactions on Automatic Control, 50, 338-355.

Wang, S., Jin, R., \& Valizadegan, H. (2010). A potential-based framework for online multi-class learning with partial feedback. In Aistat 2010. 